\title{
Review Article \\ Brain MR Contribution to the Differential Diagnosis of Parkinsonian Syndromes: An Update
}

\author{
Giovanni Rizzo, ${ }^{1,2}$ Stefano Zanigni, ${ }^{3,4}$ Roberto De Blasi, ${ }^{5}$ Daniela Grasso, ${ }^{5}$ \\ Davide Martino, ${ }^{6,7}$ Rodolfo Savica, ${ }^{8}$ and Giancarlo Logroscino ${ }^{9,10}$ \\ ${ }^{1}$ IRCCS Istituto delle Scienze Neurologiche, Bellaria Hospital, Bologna, Italy \\ ${ }^{2}$ Neurology Unit, Department of Biomedical and Neuromotor Sciences, University of Bologna, Bologna, Italy \\ ${ }^{3}$ Functional MR Unit, Policlinico S.Orsola-Malpighi, Bologna, Italy \\ ${ }^{4}$ Department of Biomedical and Neuromotor Sciences, University of Bologna, Bologna, Italy \\ ${ }^{5}$ Department of Diagnostic Imaging, Pia Fondazione di Culto e Religione "Card. G. Panico", Tricase, Italy \\ ${ }^{6}$ Department of Neurology, King's College NHS Foundation Trust, London, UK \\ ${ }^{7}$ Department of Neurology, Queen Elizabeth Hospital, Lewisham and Greenwich NHS Trust, London, UK \\ ${ }^{8}$ Department of Neurology and Health Science Research, Mayo Clinic, Rochester, MN, USA \\ ${ }^{9}$ Department of Clinical Research in Neurology, University of Bari, Pia Fondazione di Culto e Religione "Card. G. Panico", \\ Tricase, Italy \\ ${ }^{10}$ Department of Basic Medical Science, Neuroscience and Sense Organs, University of Bari, Bari, Italy
}

Correspondence should be addressed to Giovanni Rizzo; grizzo1975@libero.it

Received 22 April 2016; Revised 8 August 2016; Accepted 1 September 2016

Academic Editor: Carlo Colosimo

Copyright (c) 2016 Giovanni Rizzo et al. This is an open access article distributed under the Creative Commons Attribution License, which permits unrestricted use, distribution, and reproduction in any medium, provided the original work is properly cited.

Brain magnetic resonance (MR) represents a useful and feasible tool for the differential diagnosis of Parkinson's disease. Conventional MR may reveal secondary forms of parkinsonism and may show peculiar brain alterations of atypical parkinsonian syndromes. Furthermore, advanced MR techniques, such as morphometric-volumetric analyses, diffusion-weighted imaging, diffusion tensor imaging, tractography, proton MR spectroscopy, and iron-content sensitive imaging, have been used to obtain quantitative parameters useful to increase the diagnostic accuracy. Currently, many MR studies have provided both qualitative and quantitative findings, reflecting the underlying neuropathological pattern of the different degenerative parkinsonian syndromes. Although the variability in the methods and results across the studies limits the conclusion about which technique is the best, specific radiologic phenotypes may be identified. Qualitative/quantitative MR changes in the substantia nigra do not discriminate between different parkinsonisms. In the absence of extranigral abnormalities, the diagnosis of PD is more probable, whereas basal ganglia changes (mainly in the putamen) suggest the diagnosis of an atypical parkinsonian syndrome. In this context, changes in pons, middle cerebellar peduncles, and cerebellum suggest the diagnosis of MSA, in midbrain and superior cerebellar peduncles the diagnosis of PSP, and in whole cerebral hemispheres (mainly in frontoparietal cortex with asymmetric distribution) the diagnosis of Corticobasal Syndrome.

\section{Introduction}

Neurodegenerative parkinsonian syndromes represent a group of neurological disorders characterized by predominant motor impairment associated with nonmotor symptoms such as cognitive, psychiatric, autonomic, and sleep disorders. Idiopathic Parkinson's disease (PD) underlies the majority of cases followed by the so-called atypical parkinsonian syndromes (APSs), that is, Progressive Supranuclear Palsy (PSP), Multiple System Atrophy (MSA), and Corticobasal Syndrome (CBS). These forms have different clinical features, response to treatment, and prognosis, but clinical overlaps are frequent especially in the early stages of the disease and may lead to misdiagnosis [1]. As the definite diagnosis of these disorders requires a neuropathological confirmation and the accuracy of the clinical diagnosis is suboptimal [1], 
many studies have searched for different biomarkers able to increase the in vivo diagnostic accuracy to discriminate different parkinsonian syndromes.

Many putative biomarkers derived from genetic-epigenetic, neurophysiological, and imaging techniques have been evaluated in order to determine their diagnostic accuracy in discriminating $\mathrm{PD}$ from APSs [2]. Brain magnetic resonance $(\mathrm{MR})$ represents one of the best putative sources of biomarkers in this field, because of its relative feasibility, the absence of invasiveness, and the availability in different clinical settings.

First, brain MR imaging is useful to discriminate degenerative parkinsonian syndromes from nondegenerative forms including vascular parkinsonism, basal ganglia or thalamic tumors, normal pressure hydrocephalus, manganism, metabolic disorders such as uremic-diabetic syndrome or extrapontine myelinolysis, and inflammatory diseases [3]. Second, it may show peculiar brain alterations that are typical of different neurodegenerative diseases [3]. Third, the application of advanced neuroimaging techniques, such as morphometric-volumetric analyses, diffusion-weighted imaging (DWI), diffusion tensor imaging (DTI), tractography, magnetization transfer imaging (MTI), proton MR spectroscopy ( ${ }^{1} \mathrm{H}$-MRS), and iron-content sensitive imaging, may provide macro- and microstructural and biochemical quantitative markers of neurodegeneration in vivo. Overall, these techniques may be analysed by either voxel-wise methods that are applied to groups of subjects or regions of interest based methods that may be applied to single subjects and therefore provide information regarding brain regional degeneration. The introduction of high-field scanners $(\geq 1.5$ Tesla) has allowed a further improvement of the diagnostic potentiality of $\mathrm{MR}$, not only for research purposes but also in clinical practice, providing a better signal/noise ratio and better spatial resolution in a shorter acquisition time. This is particularly true for the iron-sensitive sequences, such as $\mathrm{T} 2{ }^{*}$-weighted and susceptibility-weighted images. High magnetic fields surely improved the advanced MR techniques, such as DTI, ${ }^{1} \mathrm{H}-\mathrm{MRS}$, and functional MRI. On the other hand, some qualitative MRI markers that are detectable by using lower field scanner may lose value using 3T or higher scanners (see below).

Our aim is to review the diagnostic usefulness of qualitative and quantitative brain MR techniques in the differential diagnosis among degenerative parkinsonian disorders. We have considered only studies that focused on the differential diagnosis between PD and APSs using methods that are readily applicable to individual patients in routine clinical practice. We did not consider the results of functional MRI studies as well as of voxel-wise analyses because of the lack of validation for the single patient use, despite their importance to pave the way to further studies focused on specific targeted regions.

\section{Qualitative Brain MRI}

Conventional MR techniques are sequences that are routinely used on a standard clinical MR scanner. Overall, these are generally qualitatively evaluated and are mainly represented by T1-weighted, T2-weighted spin-echo (SE), T2-weighted fluid-attenuated inversion-recovery (FLAIR), and proton density images. Furthermore, in recent years, sequences such as diffusion-weighted, T2*-weighted, and susceptibility-weighted images are entering into the daily clinical practice in many clinical centres. Conventional brain MRI is frequently performed in the diagnostic pipeline of a patient with parkinsonism, and it does not usually disclose clear abnormalities in PD patients. The substantia nigra (SN) involvement can be indirectly identified as a hypointensity on T2- or $\mathrm{T}^{*}{ }^{*}$-weighted images due to secondary iron deposition, but with low sensitivity and specificity. Recent studies using $3 \mathrm{~T}$ and $7 \mathrm{~T}$ MR scanners and susceptibilityweighted images (SWI) have suggested a new sign of nigral degeneration, the absence of the "swallow tail" sign [4]. This is a dorsolateral hyperintensity within the otherwise hypointense pars compacta and seems to correspond to the anatomic location of nigrosome-1, a small group of dopaminergic cells detectable in healthy individuals but not in PD patients (Figure 1) [5, 6]. However, the absence of this sign has been also reported in APSs other than $\mathrm{PD}$, providing a useful tool to discriminate parkinsonian syndromes from other diseases, for example, essential tremor or psychogenic disorders, but not PD from APSs [7, 8].

Other sequences may also be able to detect nigral neuronal loss in PD patients including a variety of inversionrecovery images [9] and a recently developed neuromelaninsensitive T1-weighted image $[10,11]$. In particular, the latter may become a useful tool in clinical practice as the visual inspection of neuromelanin-sensitive MR images by experienced neuroradiologists provides results comparable to quantitative width measurement in the detection of early stage PD SN changes [11]. As for other SN MR changes, however, neuromelanin-sensitive T1-weighted images do not represent a tool to discriminate among different forms of parkinsonian syndromes.

As concerns APSs, that is, MSA, PSP, and CBS, conventional MRI may disclose specific alterations involving further brain structures in addition to the $\mathrm{SN}$, providing radiological signs useful for the differential diagnosis [3].

MSA is clinically characterized by extrapyramidal signs accompanied by various degrees of autonomic failure, pyramidal, and cerebellar signs. Two variants have been distinguished, the cerebellar and parkinsonian types (MSA-C and MSA-P, resp.), depending on the prevalence of cerebellar or parkinsonian features. The pathological hallmark is the deposition of $\alpha$-synuclein within oligodendrocytes cytoplasm, leading to different degrees of striatonigral or olivopontocerebellar inclusions and degeneration depending on the disease subtype $[12,13]$. The MSA-P may be associated with putaminal atrophy and T2- hypointensity, mainly involving the posterior portion, and a "slit-like" marginal hyperintensity of the putamen at $1.5 \mathrm{~T}$ scans (Figure 2(a)). Unfortunately, the hyperintense putaminal rim on the T2weighted imaging on $3 \mathrm{~T}$ scans is a nonspecific finding, as it can be seen also in healthy subjects [14]. On the other hand, a putaminal hypointensity on iron-sensitive images, such as $\mathrm{T}^{*}{ }^{*}$-weighted or SWI, detected using $1.5 \mathrm{~T}$ scans and even more $3 \mathrm{~T}$ scans, helps to discriminate MSA-P from 


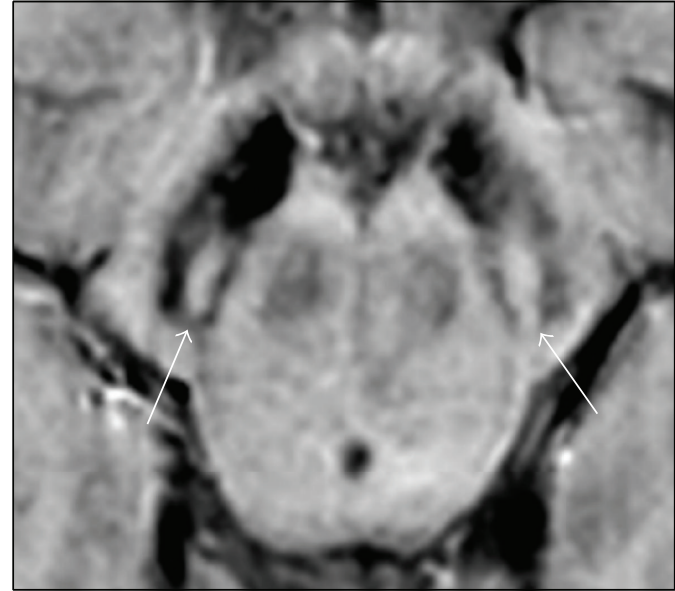

(a)

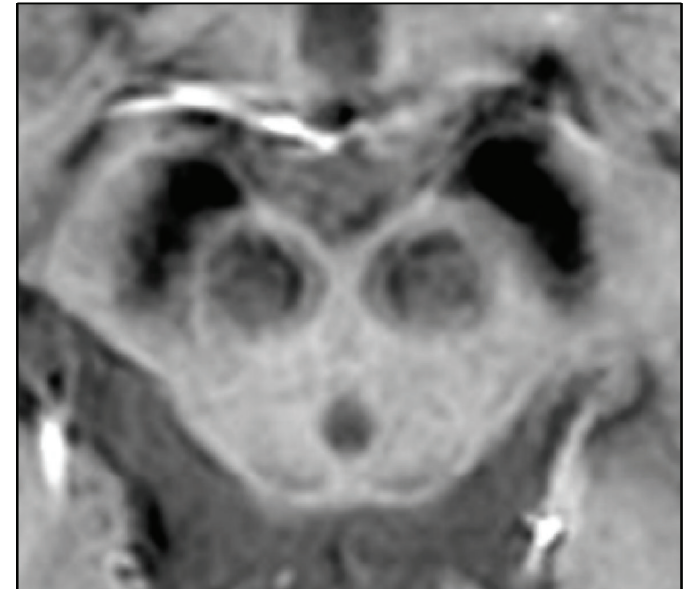

(b)

FIGURE 1: (a) High-resolution MRI (Philips Ingenia 3 T SWIp sequence) in a healthy subject: nigrosome 1 is evident as a linear, comma- or wedge-shaped hyperintense area surrounded by low signal intensity structures of the pars compacta of the substantia nigra, resembling a swallow tail (arrows). (b) In a patient with PD, nigrosome 1 is not visible bilaterally.

PD and other APSs [15-18]. As disease progresses, several radiological abnormalities of the infratentorial compartment may become apparent, that is, cerebellar atrophy, middle cerebellar peduncle (MCP) atrophy with or without $\mathrm{T} 2$ or FLAIR signal increase, pons atrophy, and a pontine cruciform T2 and proton density hyperintensity ("hot-cross bun sign") [3] (Figures 2(b)-2(f)). These infratentorial abnormalities are more frequent and earlier in MSA-C and can occur in other neurodegenerative diseases such as spinal cerebellar ataxia or fragile-X premutation syndrome [3].

PSP is mainly characterized by axial parkinsonian signs with early postural instability and falls, supranuclear gaze palsy, and poor levodopa response [19]. The neuropathological hallmarks are represented by brain tau-protein deposits, mainly within the basal ganglia and the brainstem, along with different degrees of midbrain, cerebellar, and cortical degeneration [20]. Depending on the phenotypic characteristics of onset and disease progression, PSP spectra may include different disorders such as a classical variant, the so-called Richardson's syndrome (PSP-RS), and the PSPparkinsonism variant (PSP-P), which are characterized by different clinical features depending on a differential taupathology distribution $[19,20]$.

MRI changes disclosed in PSP patients include atrophy and T2-hypointensity of putamen, mainly in the posterior portion, and of the globus pallidus, midbrain atrophy ("penguin silhouette" or "hummingbird" on sagittal images and "Mickey Mouse appearance" or "morning glory sign" on axial images), superior cerebellar peduncle (SCP) atrophy, third ventricle dilation, and periaqueductal T2-hyperintensities [3, 21] (Figure 3).

CBS represents a clinical phenotype characterized by an asymmetric, predominantly akinetic-rigid parkinsonism with poor levodopa responsiveness, limb dystonia, and higher cortical impairment represented by limb and buccolingual apraxia, alien limb phenomena, and speech disturbances [22]. Possible underlying pathologies of CBS include corticobasal degeneration, Alzheimer's disease, PSP, frontotemporal lobe degeneration, and prion disease. Putaminal atrophy and T2-hypointensity, with an asymmetric involvement, can be visible in CBS patients. Furthermore, asymmetric cortical atrophy, mainly at the level of the primary sensory-motor cortex and sometimes associated with FLAIR hyperintensity, may occur [3] (Figure 4).

All these MRI markers have good specificity but lack sensitivity, especially in the early stages. Overall, they seem to contribute little over and above the clinically based diagnosis but may be helpful when the clinical diagnosis is uncertain [23]. In particular, the presence of putaminal or infratentorial abnormalities is particularly indicative of APSs.

Finally, other than for APSs, conventional MRI is useful to diagnose other, rarer forms of degenerative parkinsonism with earlier age of onset [3]. Important examples are the marked pallidal hypointensity within a hyperintense central core of necrosis suggesting pantothenate kinase-associated neurodegeneration ("eye of the tiger") [24], T1 hyperintensities of SN and pallidum suggesting manganese accumulation [25], and T2 and FLAIR hyperintensities at the level of the basal ganglia, thalami, and brainstem ("double panda sign"), seen in Wilson's disease [26, 27].

\section{Quantitative Brain MR}

Advanced brain MR techniques represent a group of sequences and analytic methods that allow a quantitative evaluation of biochemical and macro- and microstructural alterations. The main techniques are represented by ${ }^{1} \mathrm{H}$-MRS, sequences for iron-content detection, high-resolution 3D T1-weighted sequences with morphometric and volumetric analyses, MTI, DWI, DTI, and tractography.

MRS is a noninvasive method that permits the measurement of the concentration of specific biochemical compounds in the brain in precisely defined regions guided by 


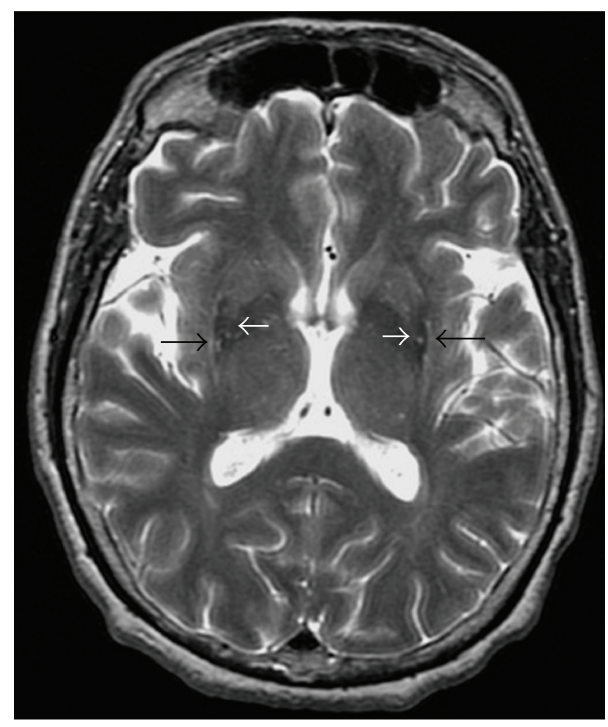

(a)

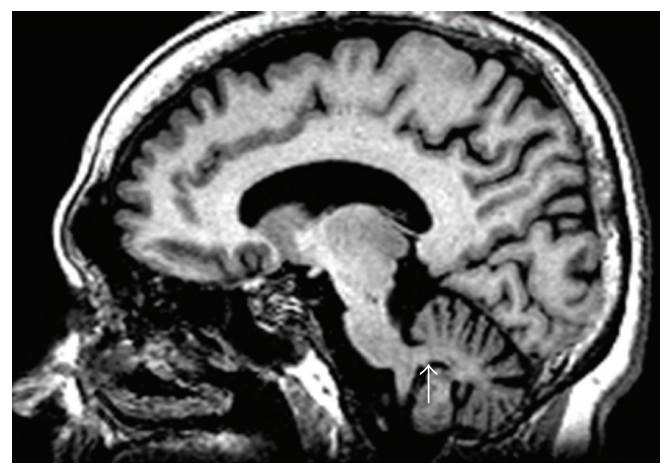

(c)

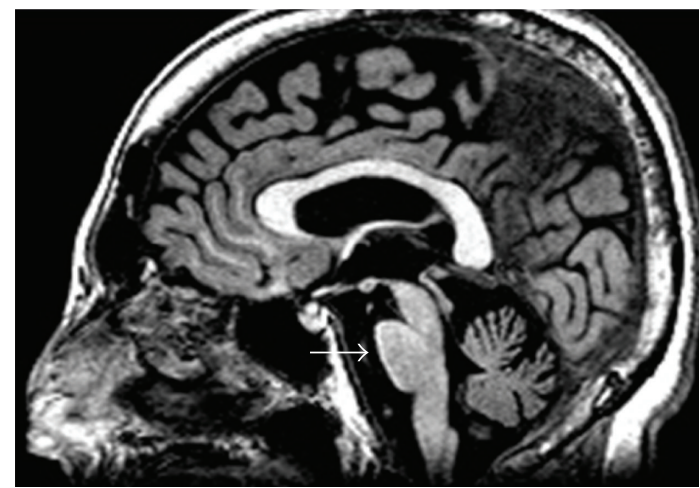

(e)

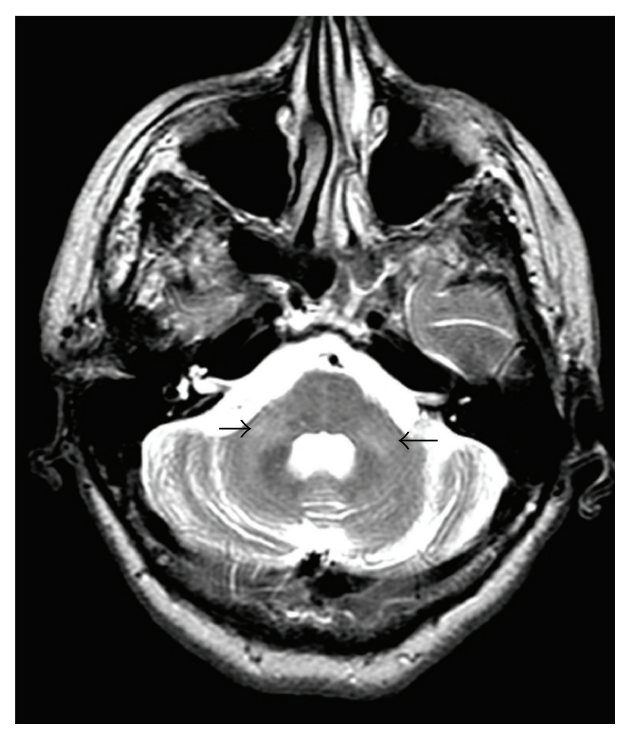

(b)

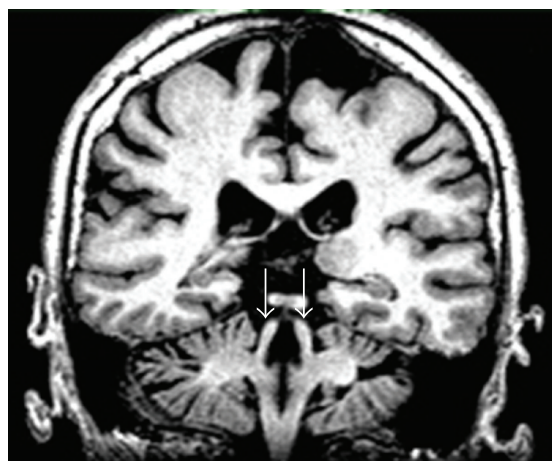

(d)

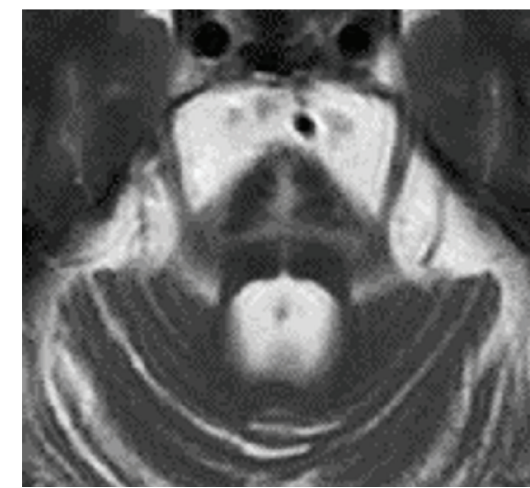

(f)

Figure 2: MRI findings in MSA. (a) Bilateral posterior putaminal T2-hypointensity (white arrows) with "slit-like" marginal hyperintensities (black arrows). (b) T2-hyperintensities of middle cerebellar peduncles (black arrows). (c) Atrophy of middle cerebellar peduncles (white arrow) on sagittal 3D T1 image. (d) Normal superior cerebellar peduncles (white arrows) on coronal 3D T1 image. (e) Pons atrophy (white arrow) on sagittal 3D T1 image. (f) Pontine cruciform T2- hyperintensity ("hot-cross bun" sign). In (b, d, e, f), cerebellar atrophy is also visible.

MRI. With MRS, spectra of many biologically important metabolites can be quantified. ${ }^{1} \mathrm{H}$-MRS is the most used in clinical practice and can detect $\mathrm{N}$-acetylaspartate- (NAA-) containing compounds (markers for neuronal integrity, viability, and number), choline-containing compounds (Cho) (major constituents of the membranes), creatinephosphocreatine $(\mathrm{Cr}$ ) (whose peak is relatively stable and commonly used as a concentration internal reference), 


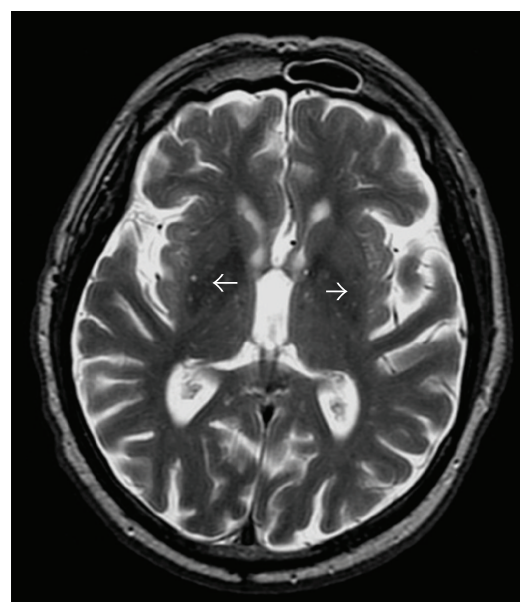

(a)

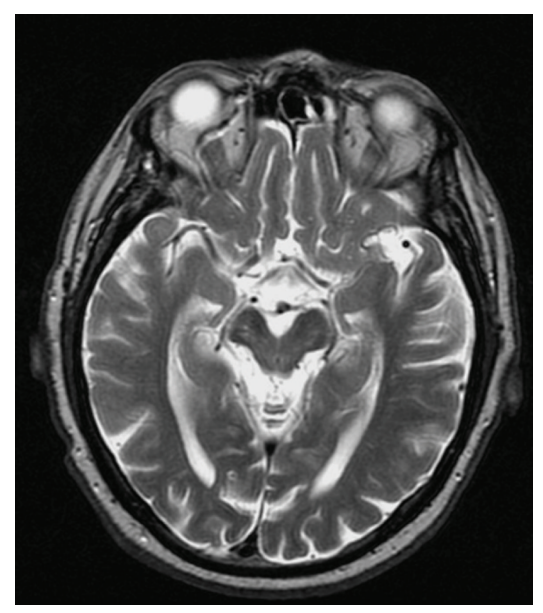

(b)

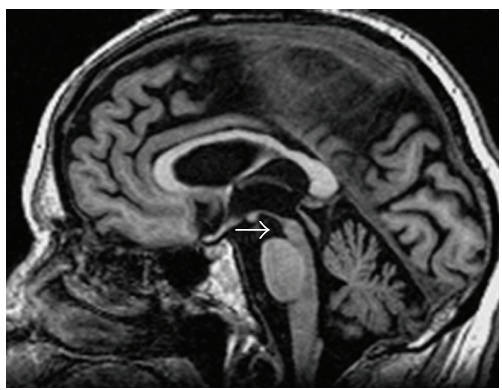

(c)

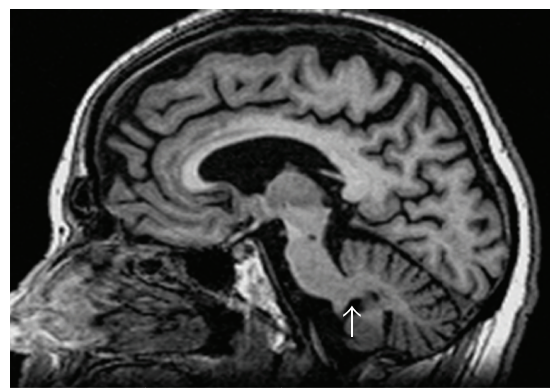

(d)

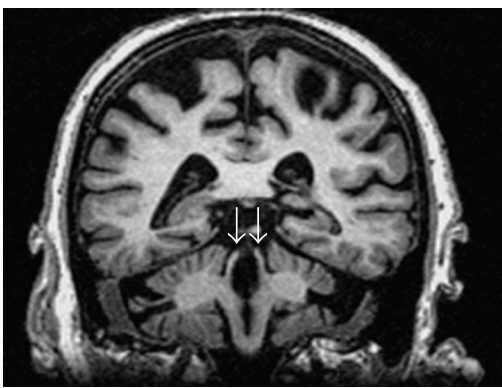

(e)

FIgURE 3: Typical MRI changes disclosed in PSP. (a) Bilateral posterior putaminal T2-hypointensity (white arrows). (b) Atrophy of the midbrain tegmentum on axial T2 image, with thinning of cerebral peduncles resulting in the concavity at the lateral margin of the midbrain, resembling Mickey Mouse or the flower morning glory. (c) Midbrain atrophy (white arrow) with a concave upper profile on sagittal 3D T1 image (brainstem profile resembling a "penguin silhouette" or a "hummingbird"). (d) Normal middle cerebellar peduncles (white arrow) on sagittal 3D T1 image. (e) Atrophy of superior cerebellar peduncles (white arrows) on coronal 3D T1 image.

glutamate and glutamine (Glx) (linked to excitatory neurotransmission), myo-inositol (mI) (a glial marker), scylloinositol (closely coupled with $\mathrm{mI}$ amount), lactate (the end product of anaerobic glycolysis), and lipids $[28,29]$.

Several MRI techniques have been used to measure nonheme iron-content in brain. The most iron-sensitive and used parameters are $\mathrm{T}^{*}$ or $\mathrm{T} 2^{\prime}$, and $\mathrm{T} 2$ to a lesser extent. Relaxometry is frequently used to evaluate the different relaxation rates $\mathrm{R} 2(1 / \mathrm{T} 2), \mathrm{R} 2^{*}\left(1 / \mathrm{T} 2^{*}\right)$, and $\mathrm{R} 2^{\prime}\left(1 / \mathrm{T} 2^{\prime}=\right.$ $\left.\mathrm{R} 2{ }^{*}-\mathrm{R} 2\right)[30]$. Further techniques include mapping of field dependent transverse relaxation rate increase (FDRI) [31], magnetic field correlation (MFC) [32], phase imaging [33], susceptibility-weighted imaging (SWI) [34], direct saturation imaging [35], and the recently developed quantitative susceptibility mapping (QSM) [36]. All these techniques provide parameters that correlate with iron content and that can be evaluated by either voxel-wise analysis methods or regions of interest based methods. However, $\mathrm{T} 2^{*}$-weighted sequences and SWI are probably the most feasible in daily clinical practice.

A number of methods are available to measure brain atrophy on high-resolution 3D T1-weighted MRI scans. These include manual morphometric measurements [37], voxel based morphometry (VBM) performing a voxel-by-voxel comparison of the density of brain gray matter and white matter across groups of subjects [38], volumetric and shape analysis of subcortical structures, and estimation of cortical thickness by using different software $[39,40]$.

DWI is sensitive to the random thermal movement of water molecules (Brownian motion) in neural tissues and is able to identify spatially resolved microstructural brain damage, via the diffusivity values (apparent diffusion coefficient, ADC; mean diffusivity, MD), which are typically elevated in brain areas where neurodegeneration occurs. DTI, based on a greater number of gradient directions and using the tensor model, allows calculating further diffusivity parameters other than MD, such as the degree of anisotropy of such diffusion, represented by fractional anisotropy (FA), axial diffusivity $(A D)$, and radial diffusivity $(\mathrm{RD})$, which are all parameters sensitive to neuronal and/or glial integrity. Overall, these sequences (DWI and DTI) may be analyzed by regions of interest or voxel-wise methods [41-43]. Another way to study DTI data is to reconstruct and evaluate the integrity of WM tracts that physically connect the different regions of the 


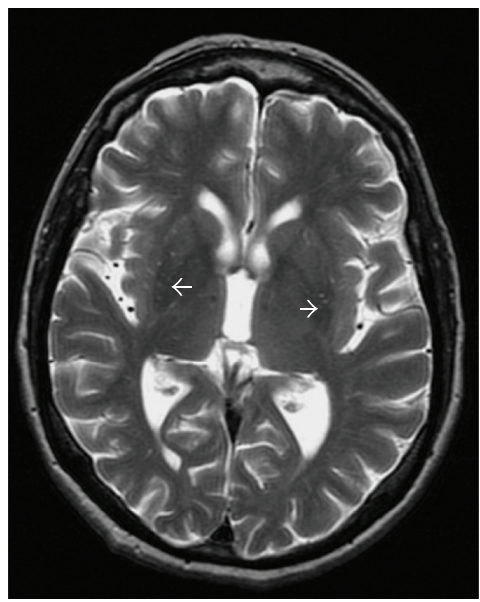

(a)

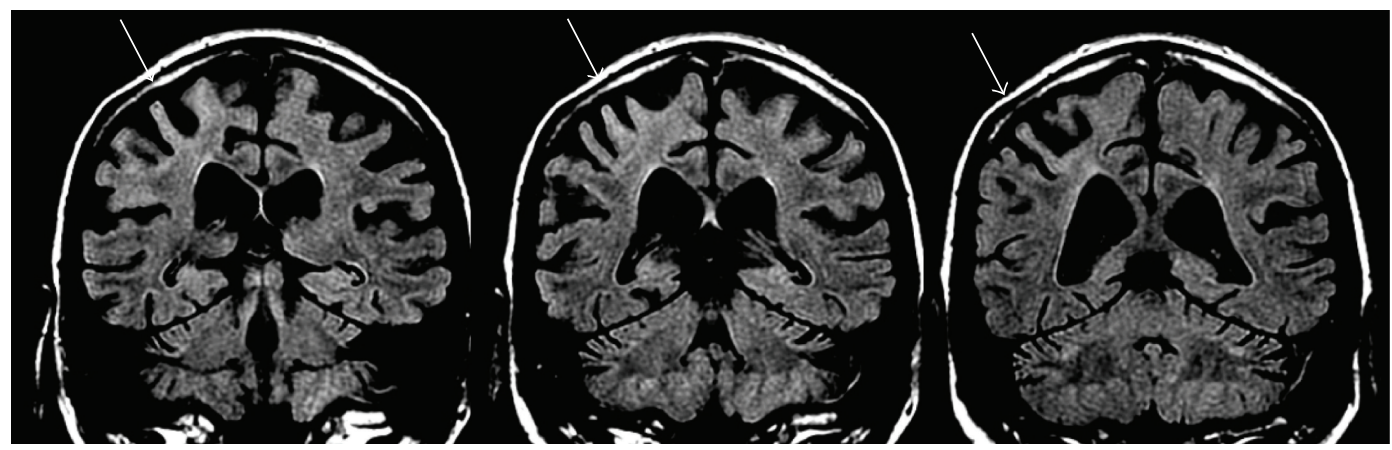

(b)

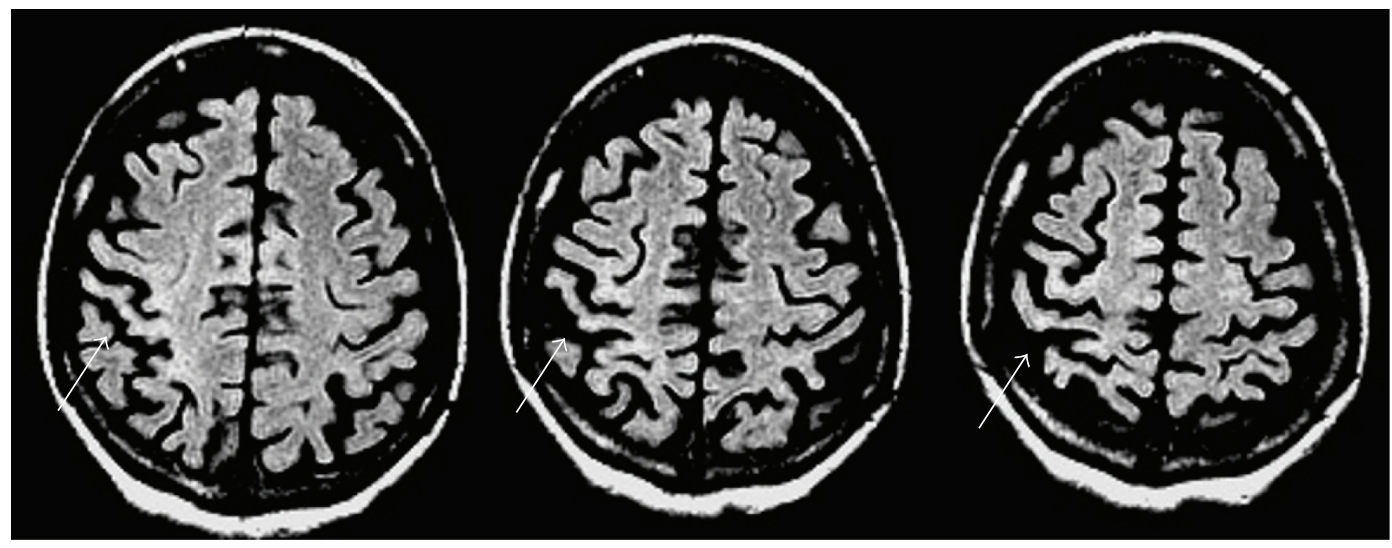

(c)

FIGURE 4: MRI changes disclosed in CBS. (a) Bilateral posterior putaminal T2-hypointensity (white arrows), mainly in the right side, where the putamen appears atrophic. (b)-(c): asymmetric cortical atrophy, mainly at the level of the right primary sensory-motor cortex associated with FLAIR hyperintensity (white arrows), on coronal (b) and axial images (c).

brain, using a number of tractography algorithms based on the anisotropic diffusion of water molecules along the axons [44].

MTI uses an off-resonance radiofrequency pulse to saturate protons in macromolecules and water molecules that are bound to macromolecules. During the pulse sequence, the saturated protons may enter the free pool of protons, primarily water, or may transfer their magnetization to free the surrounding water protons (magnetization transfer ratio, MTR), causing a decrease in the MR signal [45]. Low MTR may be caused by a reduction in the integrity of macromolecular matrix reflecting damage to the neuronal/axonal membrane or to the myelin [45].

Considering the possibility of evaluating neurodegeneration in vivo, these sequences have been applied to neurodegenerative disorders for both pathophysiological and 
diagnostic purposes, providing information regarding the different biochemical and microstructural aspects of neurodegeneration (Table 1 ).

Because of the possibility to obtain an objective quantification of regional brain alterations, these techniques may be useful in the differential diagnosis, in the follow-up, and in the response to treatment of patients with brain disorders.

The main findings provided by the studies that used quantitative brain MR techniques in patients with parkinsonian syndromes [37, 46-108] are reported in detail in Table 2, divided by the different methods applied and in Table 3 by the different brain structures studied, whereas they are briefly summarized in the following paragraphs according to the different diseases.

3.1. $P D$. The majority of studies that applied advanced MR techniques to $\mathrm{PD}$ patients provided an in vivo demonstration of neurodegenerative changes, mainly represented by neuronal loss and dysfunction, within the $\mathrm{SN}$, represented by increased iron deposition $[109,110]$, reduced neuromelanin content [10], NAA concentration [111], and altered DTI metrics $[112,113]$. Although these studies provided important information regarding PD pathophysiology and early diagnosis of degenerative parkinsonism, these alterations do not represent accurate markers for the discrimination between $\mathrm{PD}$ and APSs, reflecting the comparable SN involvement among the different diseases.

3.2. MSA. Since the presence of predominant cerebellar signs and symptoms is uncommon in $\mathrm{PD}$, the differential diagnosis with MSA-C is usually less challenging in the clinical setting. Therefore, the majority of the studies that addressed the differential diagnosis between MSA and other parkinsonisms focused on the MSA-P variant (Table 3). In particular, the main MR features related to MSA-P are represented by putaminal, pontine, MCP, and cerebellar biochemical and structural alterations compared to PD, PSP, and healthy controls (HC) (Tables 2 and 3).

Putaminal alterations indicating neuronal loss, represented by volumetric reduction, increased iron deposition, increased $\mathrm{ADC} / \mathrm{MD}$, and reduced $\mathrm{NAA}$, have all been documented as significant differences between MSA-P and PD, PSP, or HC (Tables 2 and 3). In particular, putaminal $\mathrm{ADC} / \mathrm{rTrace}(\mathrm{D}) / \mathrm{MD}$ increase and volume reduction may represent accurate markers for the discrimination of MSA$\mathrm{P}$ from $\mathrm{PD}$ with moderate-to-high sensitivity and specificity (66.7-100\% and 63.6-100\%, resp.) [61, 62, 64, 66, 68, 72, 75, $77,83,96,97]$. In addition, the increase of iron percentage in the putamen, detected by $\mathrm{T} 2{ }^{*}$-weighted sequences and SWI, is able to distinguish MSA-P from PD with moderate-to-high accuracy $(84-88 \%)[94,95]$. Similar results have also been reported in the globus pallidus and caudate (Tables 2 and 3), with high specificity (93.7\%) but lower sensitivity (62.5-75\%) in discriminating MSA-P from PD [66].

Nevertheless, because of the overall lack of specificity of basal ganglia changes, they may be useful in discriminating MSA from PD and HC but not from other APSs.

With regard to the infratentorial compartment, macroand microstructural changes mainly restricted to the pons and the MCPs, along with pontine biochemical neurodegenerative alterations, have been reported in MSA-P patients compared to PD, PSP, and HC. In particular, it has been demonstrated that a reduction of MCPs width and an increase in its ADC discriminate MSA-P from PD with high sensitivity and specificity (91-100\% and $84-100 \%$, resp.) $[48,66,67,77]$. Also increased ADC in pons and cerebellum have moderateto-high sensitivity (70\% and 60\%, resp.) and specificity $(70 \%$ and $87.5 \%$, resp.) in discriminating MSA-P from PD [75]. FA values in the caudate and RD values in MCPs have also demonstrated $90 \%$ sensitivity and $100 \%$ specificity in discriminating MSA-P from PSP [82].

In addition, cerebellar structural alterations have been reported in both MSA-P and MSA-C, including NAA reduction on MR spectroscopy (Tables 2 and 3). All these findings have a good diagnostic value allowing the discrimination between MSA and other APSs.

3.3. PSP. The majority of studies investigating the usefulness of advanced brain MR techniques in the differential diagnosis of PSP compared to PD, MSA-P, and HC focused on biochemical and macro- and microstructural alterations affecting the lenticular nucleus, midbrain, and superior cerebellar peduncles (Tables 2 and 3 ).

At a supratentorial level, the main findings are represented by the demonstration of neurodegenerative changes in the putamen and the globus pallidus, characterized by volume loss and neuronal-axonal degeneration, indicated by iron deposition, reduced NAA content, and increased water diffusivity (Tables 2 and 3). Similar results were found in the caudate nucleus and the thalamus (Tables 2 and 3). In particular, a reduction in putaminal and thalamic volumes showed a moderate-to-high accuracy (83\%), sensitivity (93\% and $73 \%$, resp.), and specificity (90\% and $70 \%$, resp.) in discriminating PSP-RS from PD [55]; pallidal volume also demonstrated a moderate-to-high accuracy (86\%) [55]. In addition, increased putaminal ADC showed moderate-tohigh sensitivity (75-90\%) and specificity (77-100\%) in discriminating PSP from PD $[62,66,70]$. Quantitative markers derived from iron-sensitive sequences demonstrated high accuracy in discriminating PSP from PD: in particular, phase shift values in the thalamus and in the pallidus (87\%) [95].

As for MSA, basal ganglia changes can discriminate PSP from $\mathrm{PD}$ and $\mathrm{HC}$ but not from other APSs. Besides the basal ganglia, diffuse structural and biochemical alterations have been demonstrated in PSP compared to PD and HC, in particular within frontal lobes, hippocampus, corpus callosum, and cingulate gyrus (Tables 2 and 3). In addition, corticospinal tract (CST) and dentatorubrothalamic tract (DRTT) microstructural alterations have been reported in PSP patients compared to PD and to PD, MSA-P, and HC, respectively $[86,88]$. However, the diagnostic utility of all these abnormalities is still uncertain. Alterations in infratentorial structures have also been demonstrated in vivo in PSP when compared to PD, MSA subtypes, and HC. In particular, atrophy and DWI/DTI metrics changes, such as increased $\mathrm{ADC}$ and $\mathrm{MD}$ and reduced FA, in midbrain and SCPs, along with alterations in the composed morphometric parameters, such as MR parkinsonism index (MRPI), midbrain/pons 
TABLE 1: Surrogate quantitative MR markers indicating different features of neurodegeneration. The main markers corresponding to the underlying pathology and the sequences needed are indicated.

\begin{tabular}{|c|c|c|c|c|}
\hline & \multicolumn{2}{|c|}{ Microstructure-biochemical profile } & \multicolumn{2}{|c|}{ Macrostructure } \\
\hline & Marker & Sequence & Marker & Sequence \\
\hline \multirow{3}{*}{$\begin{array}{l}\text { Neuronal/axonal } \\
\text { loss: degeneration }\end{array}$} & $\mathrm{ADC}$, rTrace(D), $\mathrm{MD}, \mathrm{AD}$ & DW Images & T2 signal intensity & T2-w sequences \\
\hline & NAA & ${ }^{1} \mathrm{H}-\mathrm{MRS}$ & Area diameter volume & Volumetric $\mathrm{T} 1$ sequences \\
\hline & MTR & $\begin{array}{l}\text { MTI (T1 or PD-w sequence with } \\
\text { off resonance saturation) }\end{array}$ & & \\
\hline $\begin{array}{l}\text { Glial reaction: } \\
\text { gliosis }\end{array}$ & $\mathrm{mI}$ & ${ }^{1} \mathrm{H}-\mathrm{MRS}$ & & \\
\hline \multirow{3}{*}{ Myelin disruption } & FA, RD & DW Images & T2 signal intensity & T2-w sequences \\
\hline & Cho & ${ }^{1} \mathrm{H}-\mathrm{MRS}$ & \multirow{2}{*}{ Volume } & \multirow{2}{*}{ Volumetric $\mathrm{T} 1$ sequences } \\
\hline & MTR & $\begin{array}{l}\text { MTI (T1 or PD-w sequence with } \\
\text { off resonance saturation) }\end{array}$ & & \\
\hline \multirow{2}{*}{ Iron content } & $\mathrm{T} 2 * / \mathrm{R} 2 *$ values & $\mathrm{T} 2^{*} / \mathrm{R} 2^{*}-\mathrm{w}$ sequence & & \\
\hline & Phase shift values & SWI phase images & & \\
\hline
\end{tabular}

ratio, and MCP/SCP ratio, seem to be the markers with the best accuracy in discriminating PSP from other parkinsonisms (Tables 2 and 3). Overall, these markers showed a higher accuracy in discriminating PSP from other diseases compared to markers derived from supratentorial structures. In particular, the most accurate markers are represented by midbrain area (95-100 sensitivity and 91.3-98\% specificity) [47, 49, 54], SCP diameter (65.5\% sensitivity and $93.5 \%$ specificity) [49], MCP/SCP ratio (78.8\% sensitivity and $88.9 \%$ specificity) [37], pons/midbrain ratio (90-96\% sensitivity and 90-96\% specificity) $[37,51,54]$, midbrain/pons ratio (63.6$100 \%$ sensitivity and $92.1 \%$ specificity) $[47,50]$, and MRPI (81.8-100\% sensitivity and $80.2-100 \%$ specificity) [37, 50$52,54]$. Increased MD in SCPs and in the posterior fossa showed moderate accuracy in discriminating PSP-RS from PD (83\%) [54].

3.4. CBS. Studies focusing on the basal ganglia found biochemical (reduced NAA content $[101,103]$ ) and microstructural (increased diffusivity [70]) putaminal changes in CBS, which may be helpful in discriminating it from PD and HC but not from other APSs. Volume loss in the thalamus was found in CBS versus PD and HC, but similar to PSP [84], whereas increased diffusivity in different thalamic regions seems to discriminate CBS from PSP [78].

Taking into account that cortical impairment represents a hallmark of CBS, most of the studies focused on the application of advanced MR techniques to the supratentorial compartment, providing findings that are more helpful. In particular, asymmetric atrophy in frontal, parietal, and occipital lobes indicated by a volumetric study [57] along with microstructural alterations, represented by increased diffusivity in the whole cerebral hemispheres, asymmetrically [70] or in the precentral and postcentral gyri [88], have been reported in CBS patients compared to PD, PSP, and HC. In addition, reduced frontoparietal cortex NAA content $[101,103]$ and morphometric [57] and DTI changes [80] of the corpus callosum have also been demonstrated in CBS compared to $\mathrm{PD}$ and $\mathrm{HC}$, but with few data about the discriminating value compared to the other APSs.

\section{Discussion}

Currently, a plethora of MR studies focusing on diagnostic markers in parkinsonian syndromes is available in the literature. Earlier studies have highlighted the qualitative MRI changes found in the different atypical parkinsonian syndromes that reflect the underlying neuropathological pattern; these changes showed good specificity but low sensitivity. More recently, several quantitative methods have been introduced in the attempt to increase the sensitivity of MR findings. Despite their promising results, however, the variability of the methods used is still a limitation to their widespread application. Moreover, there is a wide variability of results across studies using the same methodology, which does not allow drawing firm conclusions on the applicability of most of the methods used.

Although structural and biochemical alterations in different brain regions have been reported to have a high diagnostic accuracy, studies evaluating these different features in the same population are few $[53,54,83,84,88,96]$. Therefore, a comparison of the diagnostic properties of different $\mathrm{MR}$ markers is difficult. Furthermore, the potential and the results of each technique depend on magnetic fields, resolution, type of pre- and postprocessing, and statistical analysis adopted, possibly affecting the reproducibility of the findings. The image-processing pipeline is crucial to obtain reliable data and can be very long and full of pitfalls (e.g., as regards DTI, susceptibility correction, eddy current correction, head motion correction, estimate of the tensor in each voxel, and extraction of quantitative parameters) [114], and each step is susceptible to sources of bias, which may not only limit the accuracy and precision but can lead to a misinterpretation of the results. Other than technical considerations, a limitation of most of the studies analyzing manually segmented structures is the operator-dependence. Different MRI studies 


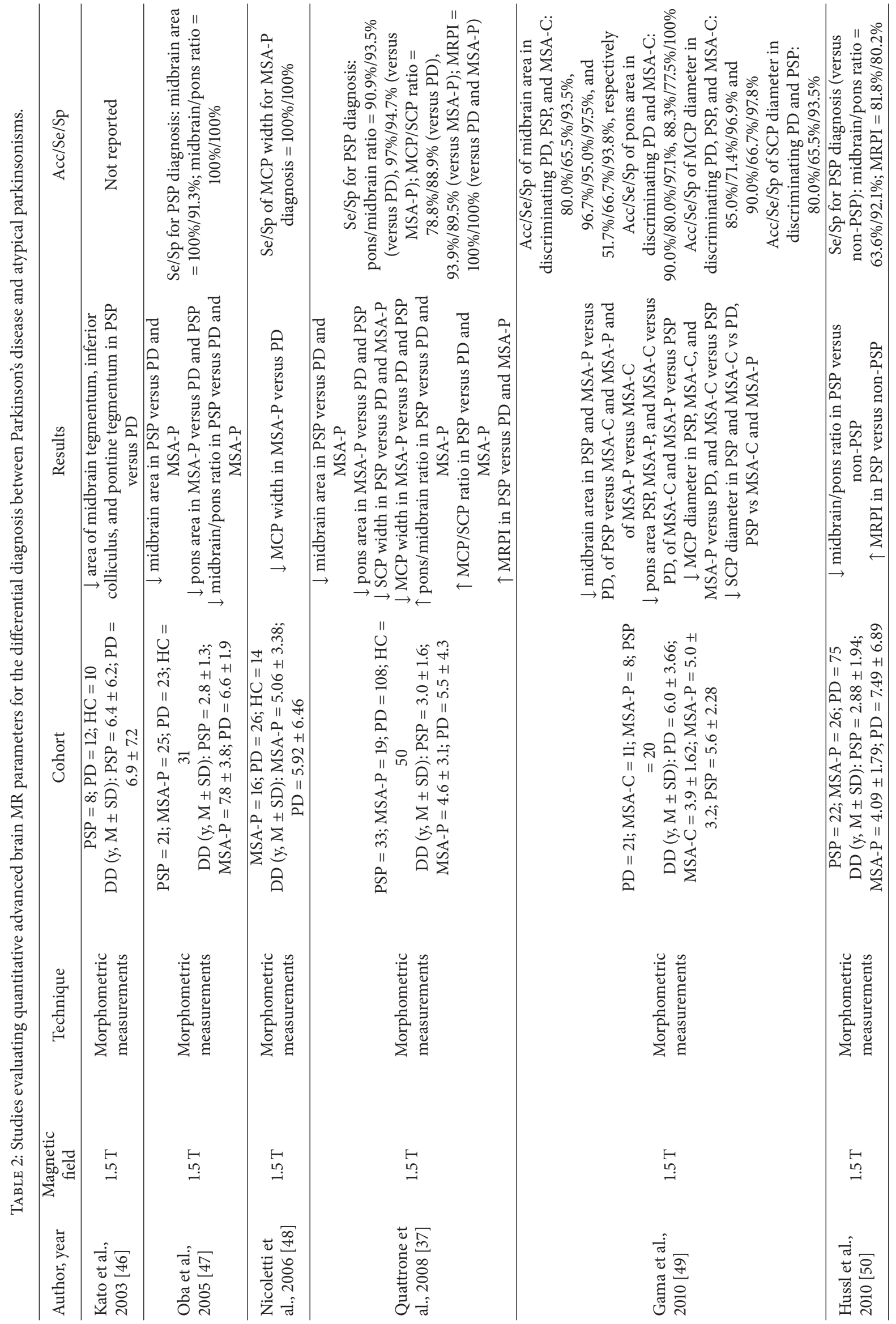




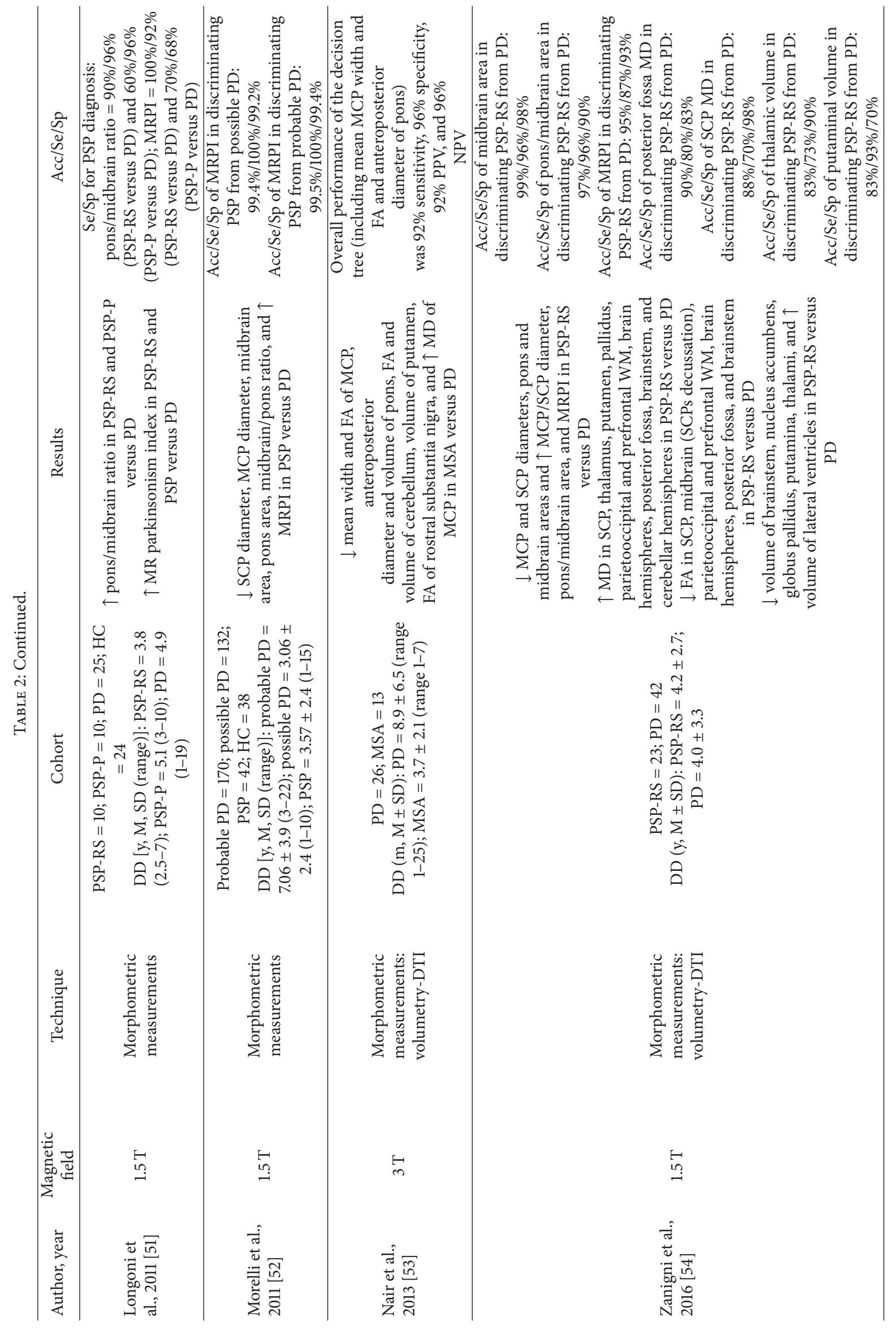




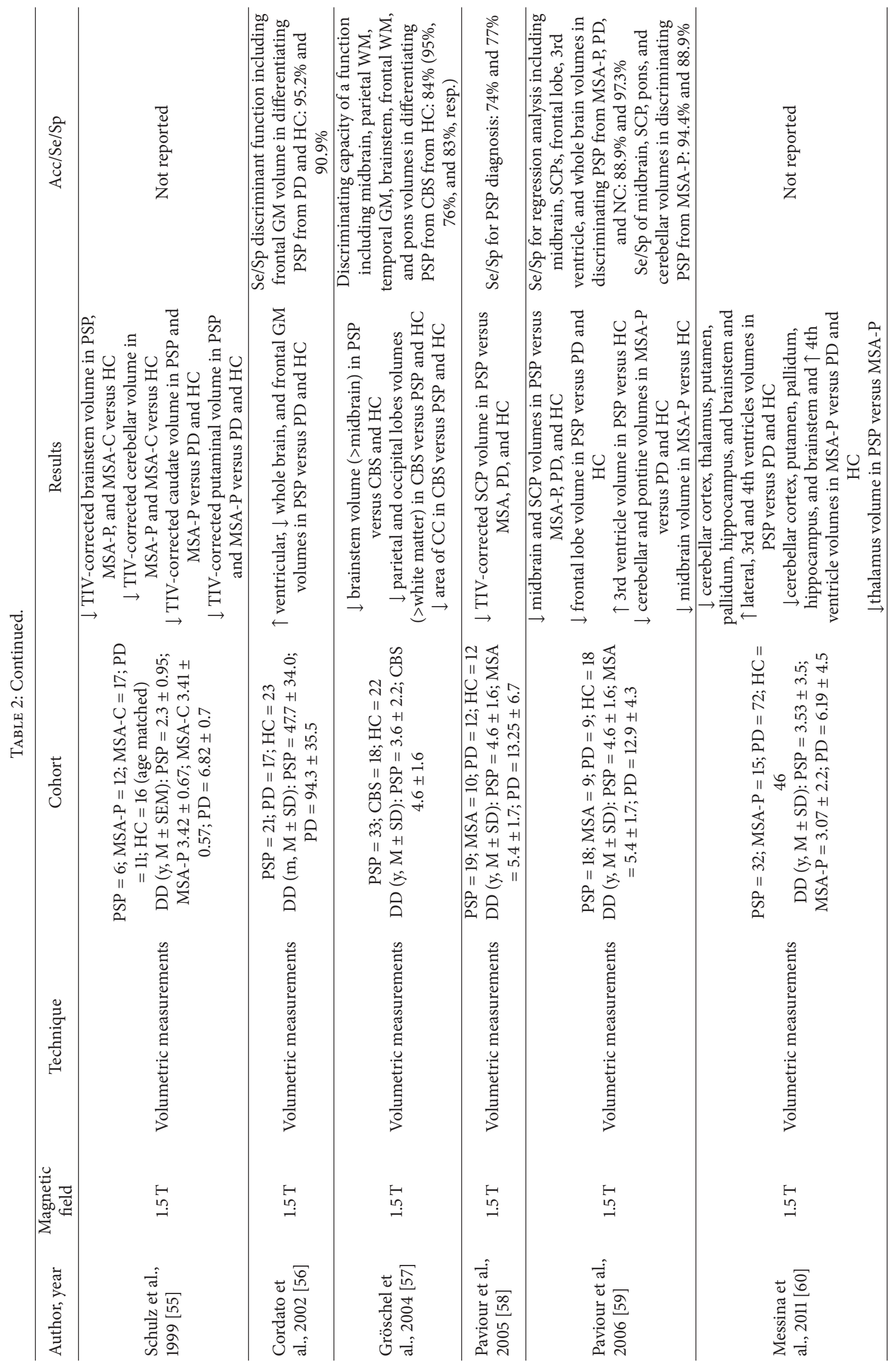




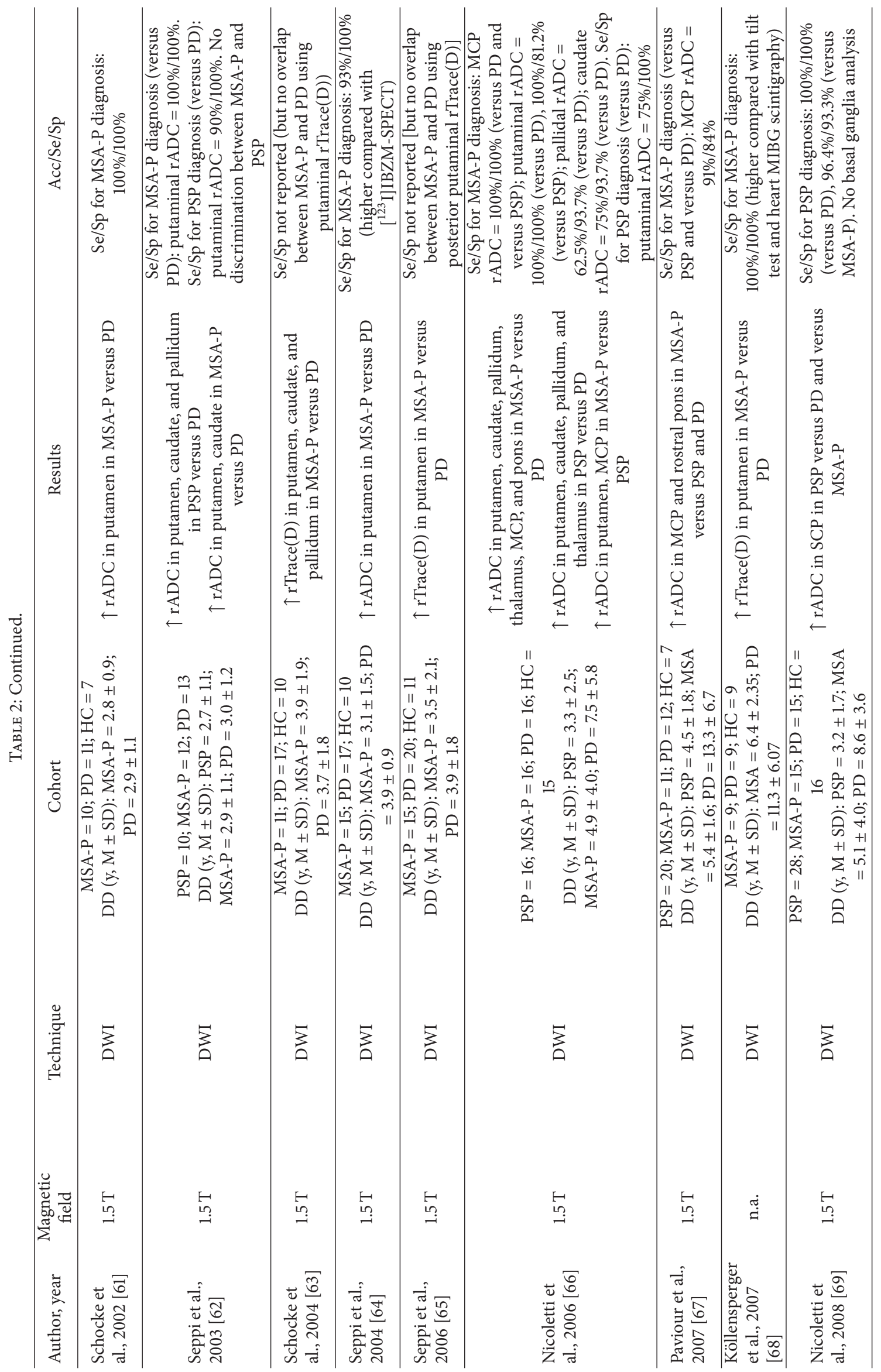




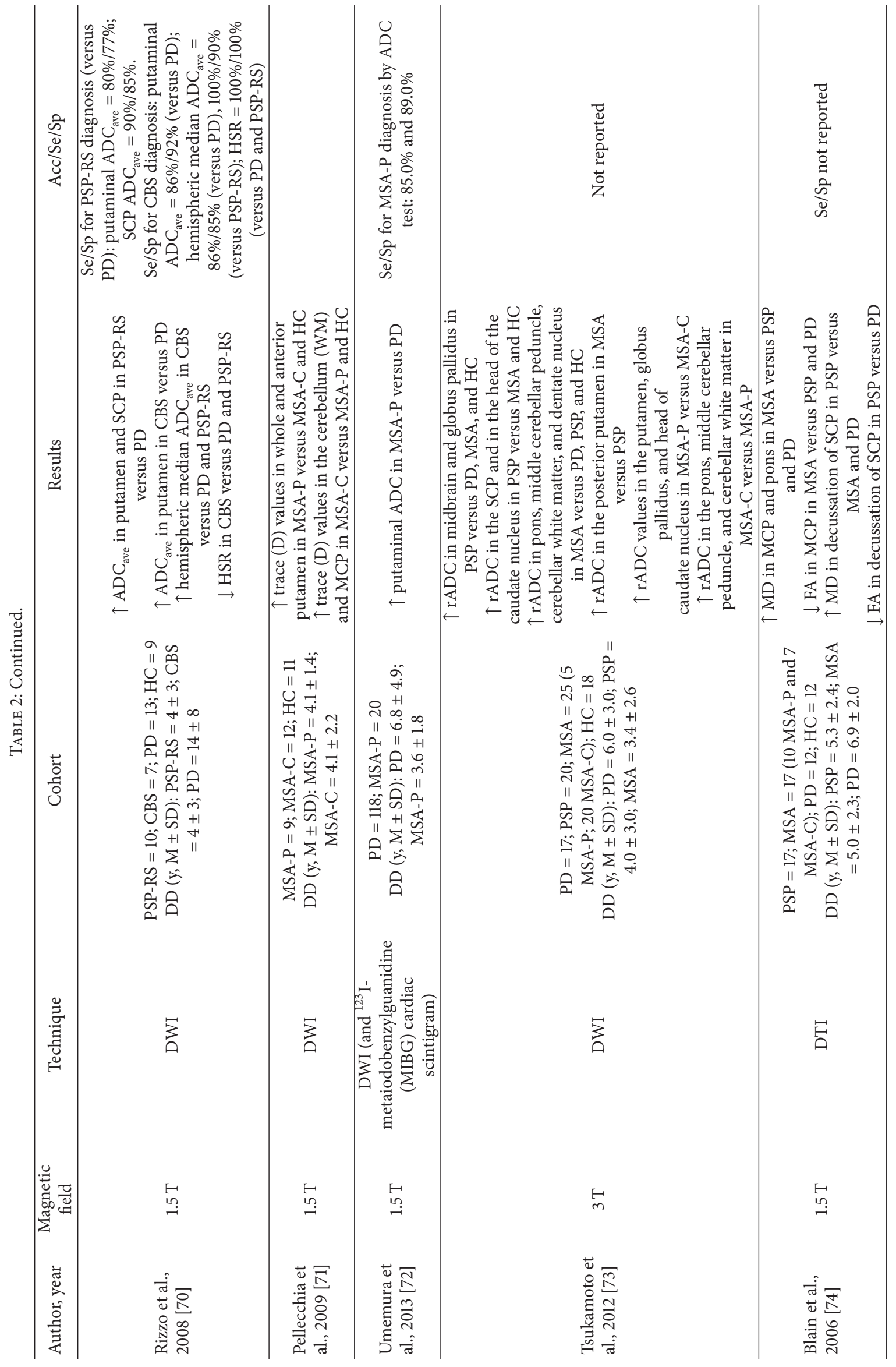




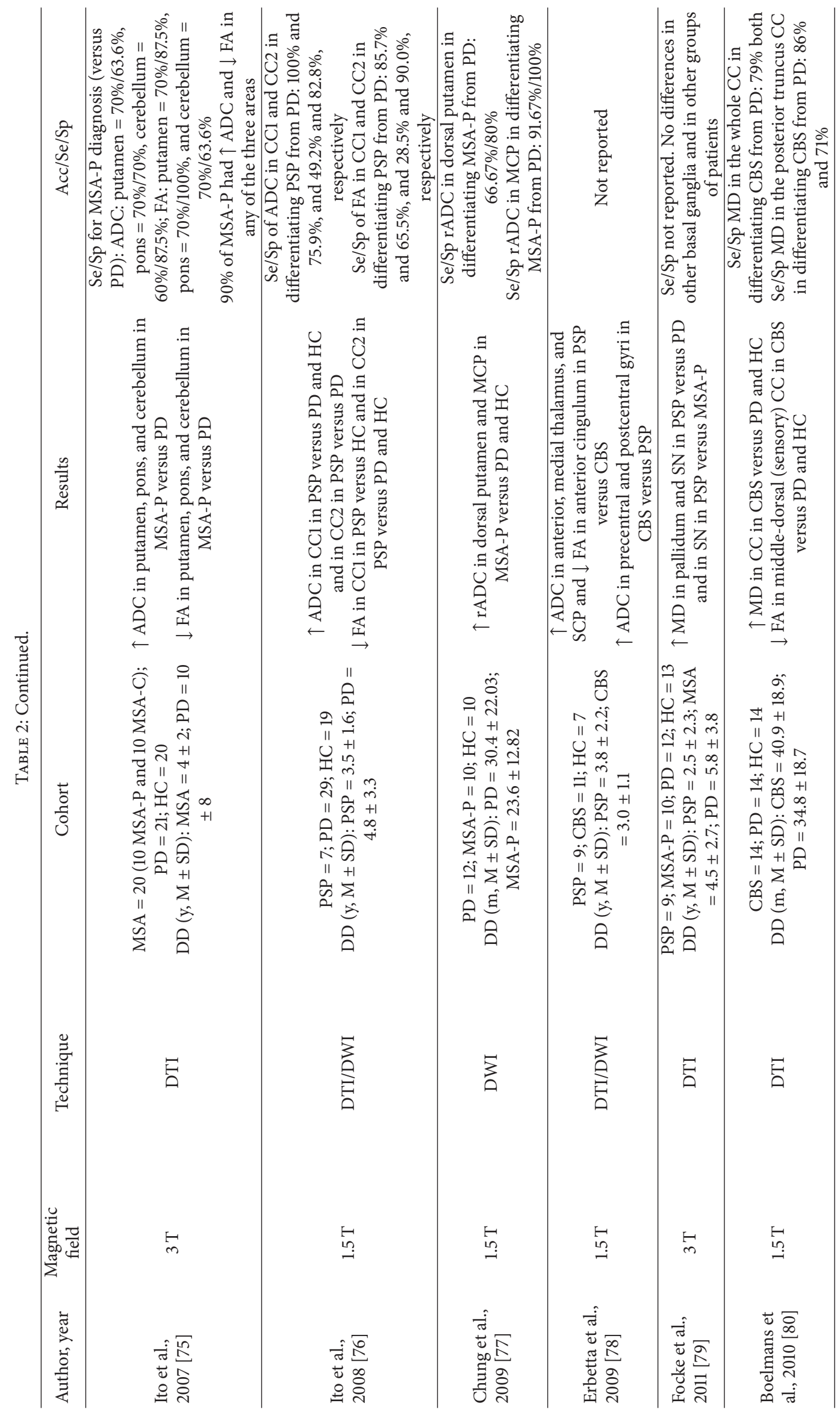




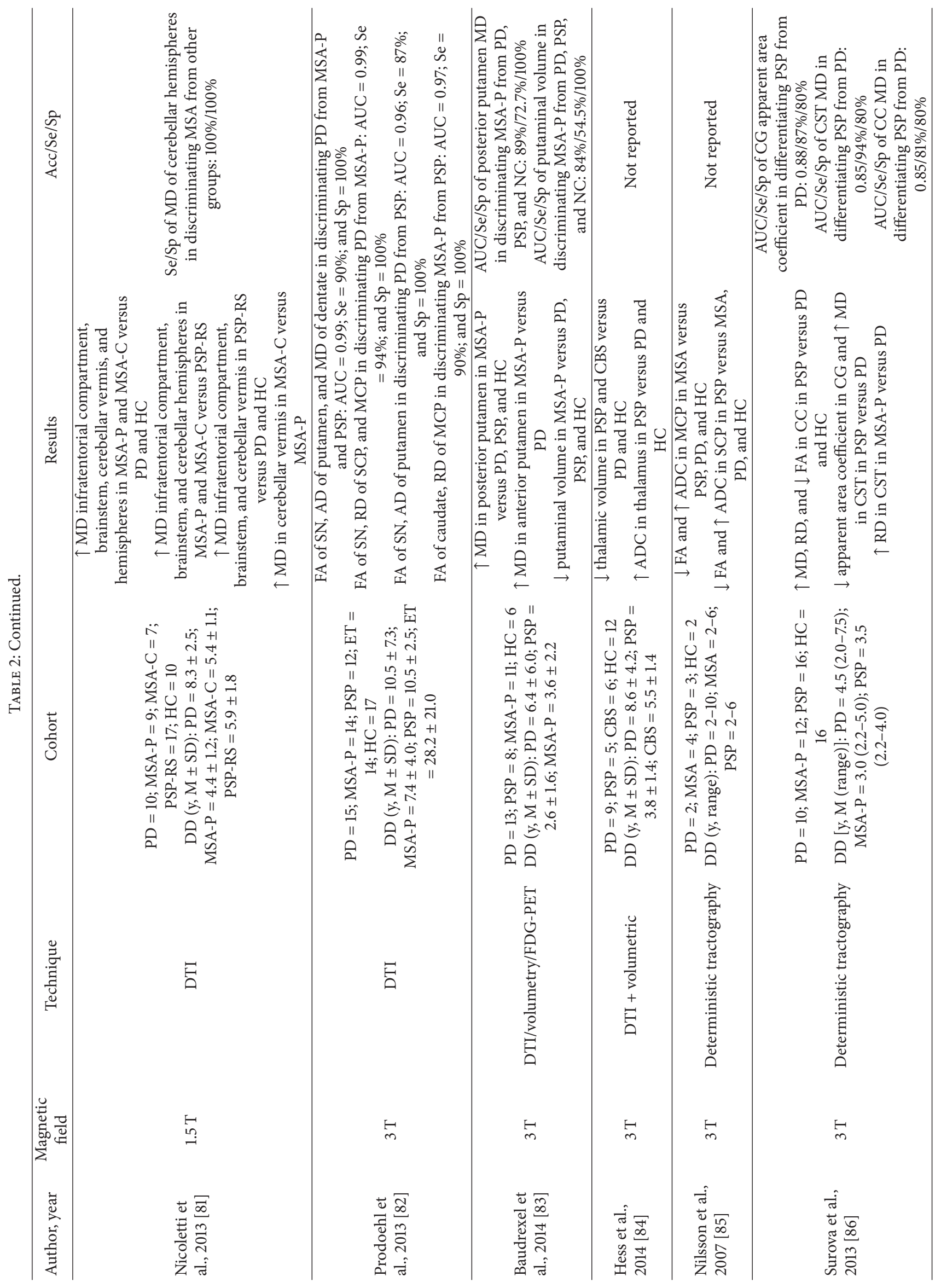




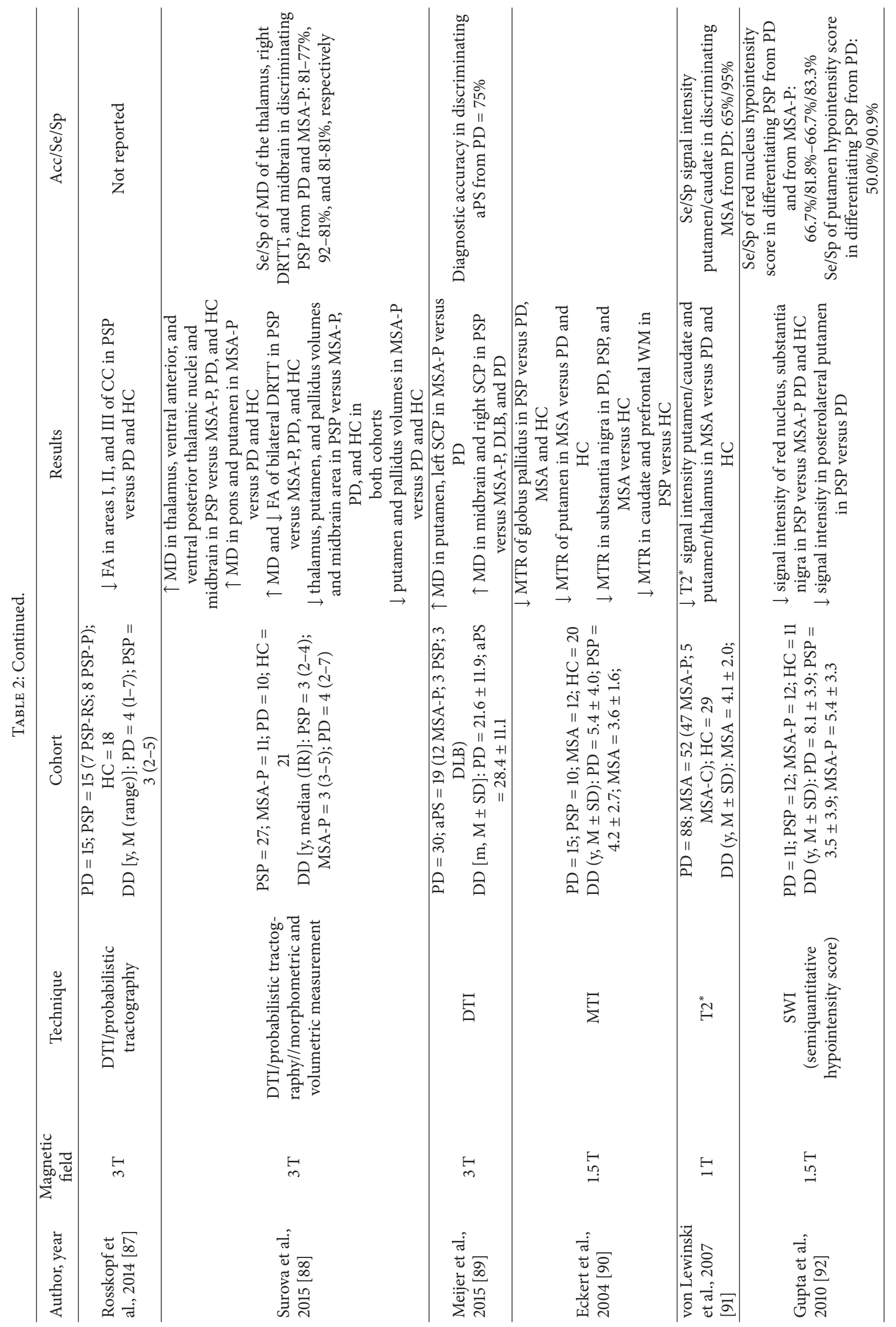




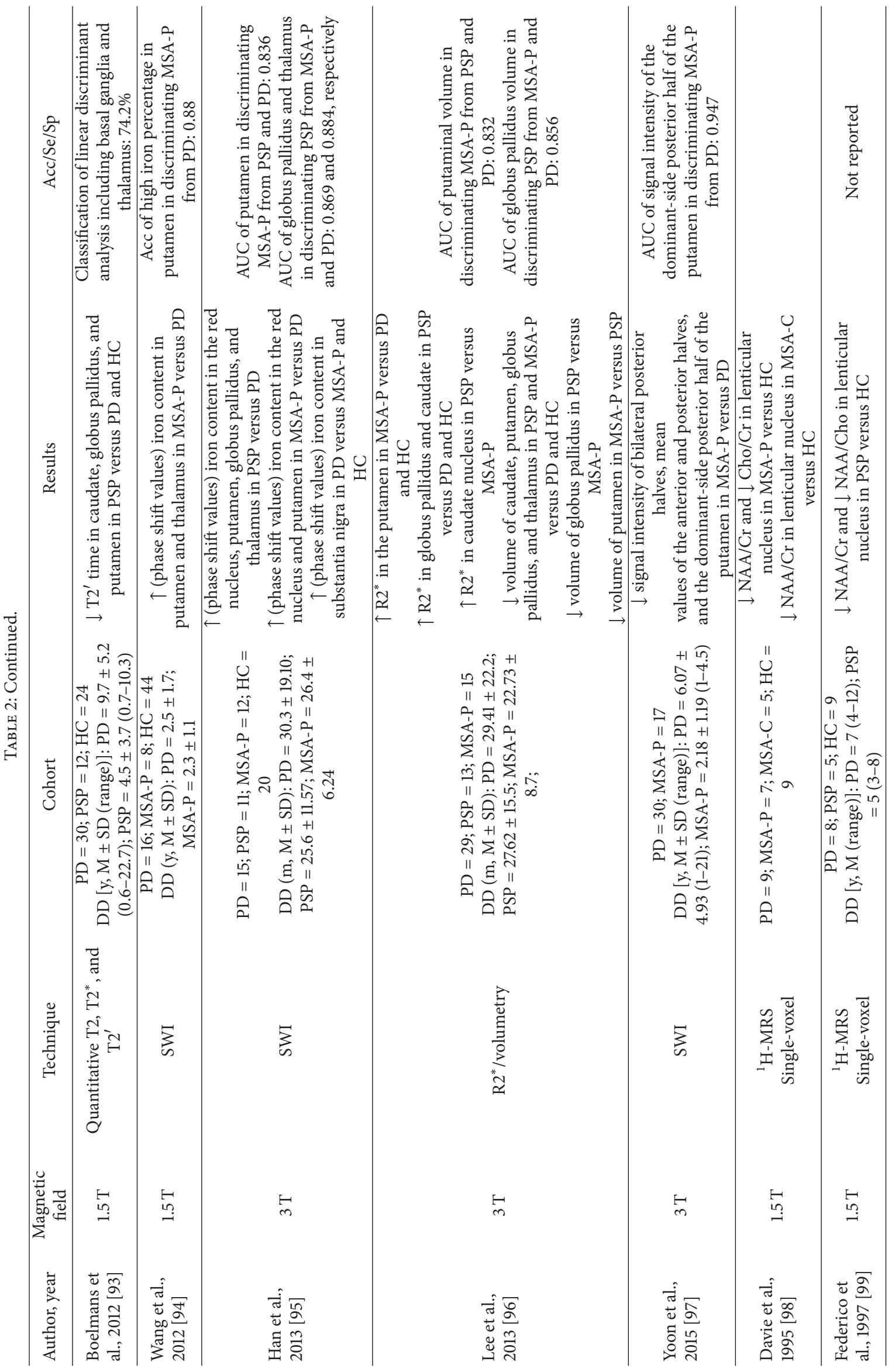




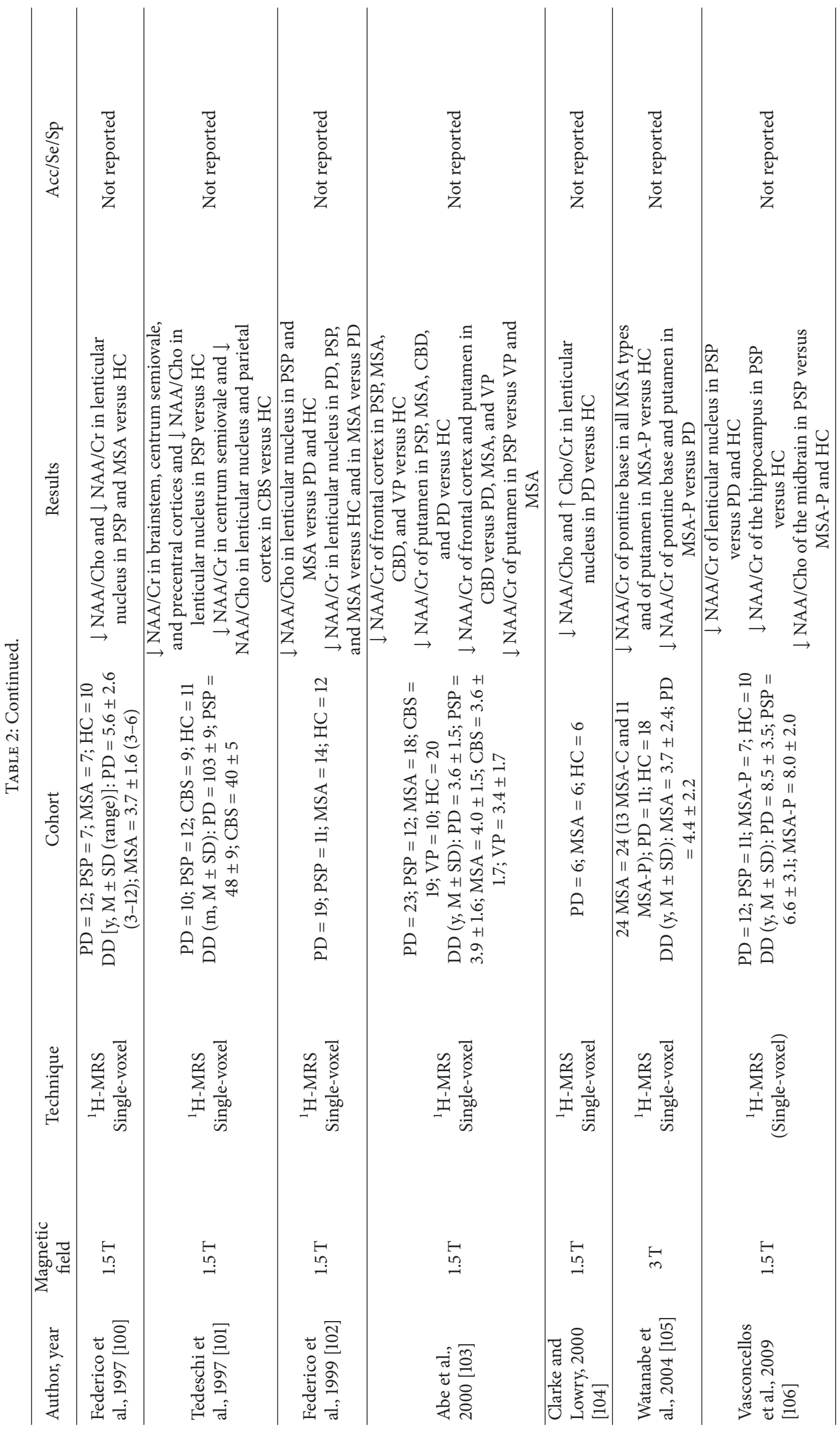




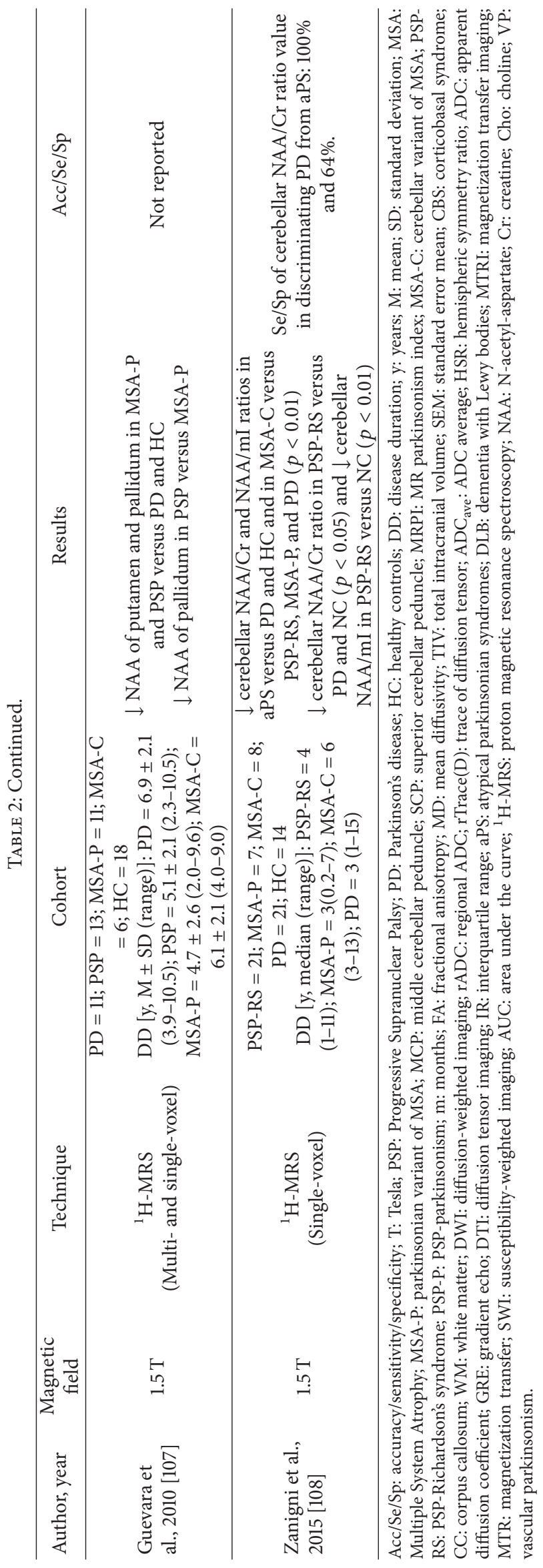


TABLE 3: Anatomical distribution of brain alterations in multiple system atrophy (MSA), in the cerebellar (-C) and parkinsonian (-P) MSA variants, in progressive supranuclear palsy (PSP), and in corticobasal syndrome (CBS) (results of those studies focused on the differential diagnosis between Parkinson's disease and atypical parkinsonian syndromes).

\begin{tabular}{|c|c|c|c|c|c|}
\hline & MSA & MSA-C & MSA-P & PSP & CBS \\
\hline \multicolumn{6}{|c|}{ Basal ganglia } \\
\hline Putamen & $\begin{array}{c}\downarrow \text { volume }{ }^{*}[53] \\
\uparrow \mathrm{ADC}^{\wedge}[73] \\
\downarrow \mathrm{MTR}^{* \dagger}[90] \\
\downarrow \mathrm{T}^{*} \text { signal } \\
\text { intensity } \\
\downarrow \mathrm{NAA}^{* \dagger} \mathrm{Cr}^{\dagger}[101]\end{array}$ & & $\begin{array}{c}\downarrow \text { volume } \mathrm{e}^{* \wedge \dagger} \\
{[55,60,83,86,96]} \\
\uparrow \mathrm{ADC}^{* \circ \S \dagger}[61,62,64, \\
66,73,75,77] \\
\uparrow \mathrm{rTrace}(\mathrm{D})^{* \S \dagger} \\
{[63,65,68,71]} \\
\downarrow \mathrm{FA}^{*}[75] \\
\uparrow \mathrm{MD}^{* \wedge \dagger}[83,86,89] \\
\uparrow \text { phase shift values }^{*} \\
{[94,95]} \\
\uparrow \mathrm{R}^{*} \text { signal } \\
\text { intensity }{ }^{* \dagger}[96] \\
\downarrow \text { SWI signal } \\
\text { intensity* }[97] \\
\downarrow \text { NAA/Cr*广 }[105] \\
\downarrow \text { NAA }{ }^{* \dagger}[107]\end{array}$ & $\begin{array}{c} \\
\downarrow \text { volume }^{* \dagger \dagger} \\
{[54,55,60,86,96]} \\
\uparrow \\
\mathrm{ADC}^{*}[62,66,70,72] \\
\downarrow \mathrm{T}^{\prime} \text { time }^{* \dagger}[93] \\
\uparrow \mathrm{MD}^{*}[54] \downarrow \text { SWI } \\
\text { signal intensity }{ }^{*}[92] \\
\uparrow \text { phase shift values } \\
{[95]} \\
\downarrow \mathrm{NAA}^{*} \mathrm{Cr}^{\# \dagger}[103] \downarrow \\
\mathrm{NAA}^{* \dagger}[107]\end{array}$ & $\begin{array}{c}\uparrow \mathrm{ADC}^{*}[70] \\
\downarrow \mathrm{NAA}^{*} \mathrm{Cr}^{* \# \uparrow}[103]\end{array}$ \\
\hline Globus pallidus & & & $\begin{array}{c}\downarrow \text { volume } e^{* \dagger} \\
{[60,86,96]} \\
\uparrow \operatorname{rTrace}(\mathrm{D})^{*}[63] \\
\uparrow \mathrm{ADC}^{* \S}[66,73] \\
\downarrow \operatorname{NAA}^{* \dagger}[107]\end{array}$ & $\begin{array}{c}\downarrow \text { volume } \\
{[54,60,86,96]} \\
\uparrow \mathrm{ADC}^{* \dagger \dagger}[62,66,73] \\
\uparrow \mathrm{MD}^{*}[54,79] \\
\downarrow \mathrm{MTR}^{* \# \dagger}[90] \\
\downarrow \mathrm{T}^{\prime} \text { time } \\
\text { *† }[93] \\
\uparrow \text { phase shift values* } \\
{[95]} \\
\uparrow \mathrm{R}^{*} \text { signal } \\
\text { intensity }{ }^{* \dagger}[96] \\
\downarrow \mathrm{NAA}^{* \circ \dagger}[107]\end{array}$ & \\
\hline Lenticular nucleus & $\begin{array}{c}\downarrow \mathrm{NAA} / \mathrm{Cr}^{* \dagger}[100,102] \\
\downarrow \mathrm{NAA}^{*} \mathrm{Cho}^{* \dagger} \\
{[100,102]}\end{array}$ & $\downarrow \mathrm{NAA} / \mathrm{Cr}^{\dagger}[98]$ & $\begin{array}{l}\downarrow \mathrm{NAA} / \mathrm{Cr}^{\dagger}[98] \\
\downarrow \mathrm{Cho}^{\dagger} \mathrm{Cr}^{\dagger}[98]\end{array}$ & $\begin{array}{c}\downarrow \mathrm{NAA} / \mathrm{Cr}^{* \dagger} \\
{[99-102,109]} \\
\downarrow \mathrm{NAA} / \mathrm{Cho}^{* \dagger} \\
{[99,100,102]}\end{array}$ & $\downarrow \mathrm{NAA} \mathrm{Cho}^{\dagger}[101]$ \\
\hline Caudate & & & $\begin{array}{c}\downarrow \text { volume }^{* \dagger}[55,96] \uparrow \\
\operatorname{ADC}^{* \S}[62,66,73] \\
\uparrow \operatorname{rTrace}(D)^{*}[63]\end{array}$ & $\begin{array}{c}\downarrow \text { volume }^{* \dagger}[55,96] \uparrow \\
\mathrm{ADC}^{* \# \dagger}[62,66,73] \\
\downarrow \mathrm{MTR}^{\dagger}[90] \\
\downarrow \mathrm{T}^{\prime} \text { time }^{* \dagger}[93] \\
\uparrow \mathrm{R}^{*} \text { signal } \\
\text { intensity } \\
\text { in }[96]\end{array}$ & \\
\hline Nucleus accumbens & & & & $\downarrow$ volume $^{*}[54]$ & \\
\hline Thalamus & & & $\begin{array}{c}\uparrow \text { ADC }^{*}[66] \\
\uparrow \text { phase shift values* } \\
{[94]} \\
\downarrow \text { volume* }\end{array}$ & $\begin{array}{c}\downarrow \text { volume }^{* \circ \dagger} \\
{[54,60,84,86,96]} \\
\uparrow \mathrm{ADC}^{* \lambda \dagger}[66,78] \\
\uparrow \mathrm{MD}^{* \dagger \dagger}[54,86] \\
\uparrow \text { phase shift values* } \\
{[95]} \\
\end{array}$ & $\downarrow$ volume $^{* \dagger}[84]$ \\
\hline \multicolumn{6}{|c|}{ Supratentorial compartment } \\
\hline Whole brain & & & & $\begin{array}{c}\downarrow \text { volume }^{* \dagger}[56] \\
\uparrow \mathrm{MD}^{*}[54] \\
\downarrow \mathrm{FA}^{*}[54]\end{array}$ & $\begin{array}{l}\uparrow \mathrm{ADC}^{* \wedge}[70] \\
\downarrow \mathrm{HSR}^{* \wedge}[70]\end{array}$ \\
\hline Frontal lobe & $\downarrow \mathrm{NAA} / \mathrm{Cr}^{\dagger}[103]$ & & & $\begin{array}{c}\downarrow \text { volume }{ }^{* \dagger}[56,59] \\
\uparrow \mathrm{MD}^{*}[54] \\
\downarrow \mathrm{FA}^{*}[54] \\
\downarrow \mathrm{MTR}^{\dagger}[90] \\
\downarrow \text { precentral cortices } \\
\text { NAA/Cr }{ }^{\dagger}[101] \\
\downarrow \text { NAA/Cr }{ }^{\dagger}[103]\end{array}$ & $\begin{array}{c}\uparrow \text { ADC precentral } \\
\text { gyrus }^{\wedge}[78] \\
\downarrow \mathrm{NAA}^{\mathrm{NAC}} \mathrm{Cr}^{* \#+}[103]\end{array}$ \\
\hline
\end{tabular}


TABle 3: Continued.

\begin{tabular}{|c|c|c|c|c|c|}
\hline & MSA & MSA-C & MSA-P & PSP & CBS \\
\hline Parietal lobe & & & & $\begin{array}{l}\uparrow \mathrm{MD}^{*}[54] \\
\downarrow \mathrm{FA}^{*}[54]\end{array}$ & 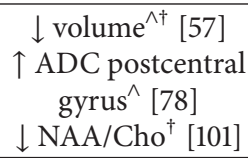 \\
\hline Occipital lobe & & & & & $\downarrow$ volume $^{\wedge \dagger}[57]$ \\
\hline Corpus Callosum & & & & $\begin{array}{c}\uparrow \mathrm{ADC}^{\mathrm{A}} \text { in } \mathrm{CCl}^{* \dagger} \text { and } \\
\mathrm{CC} 2^{*}[76] \\
\downarrow \mathrm{FA} \text { in } \mathrm{CC}^{\dagger} \text { and } \\
\mathrm{CC}^{* \dagger}[76] \\
\uparrow \mathrm{MD}^{* \dagger}[86] \\
\uparrow \mathrm{RD}^{* \dagger}[86] \\
\downarrow \mathrm{FA}^{* \dagger}[86,87]\end{array}$ & $\begin{array}{l}\downarrow \operatorname{area}^{\wedge \dagger}[57] \\
\uparrow \mathrm{MD}^{* \dagger}[80] \\
\downarrow \mathrm{FA}^{* \dagger}[80]\end{array}$ \\
\hline Cingulate gyrus & & & & $\begin{array}{c}\uparrow \mathrm{ADC}^{\lambda} \text { anterior CG } \\
{[78]} \\
\downarrow \text { apparent area } \\
\text { coefficient }^{*}[86]\end{array}$ & \\
\hline Hippocampus & & & $\downarrow$ volume $^{* \dagger}[60]$ & $\begin{array}{c}\downarrow \text { volume }^{* \dagger}[60] \\
\downarrow \text { NAA/Cr } \\
\end{array}$ & \\
\hline Centrum semiovale & & & & $\downarrow \mathrm{NAA} / \mathrm{Cr}^{\dagger}[101]$ & \\
\hline Lateral ventricles & & & & $\begin{array}{l}\uparrow \text { volume }^{* \dagger} \\
{[54,56,60]}\end{array}$ & \\
\hline $3 r d$ ventricle & & & & $\uparrow$ volume $^{* \dagger}[59,60]$ & \\
\hline 4th ventricle & & & $\uparrow$ volume $^{* \dagger}[60]$ & $\uparrow$ volume $^{* \dagger}[60]$ & \\
\hline \multicolumn{6}{|c|}{ White matter tracts } \\
\hline CST & & & $\uparrow \mathrm{RD}[86]$ & $\uparrow \mathrm{MD}^{*}[86]$ & \\
\hline$D R T T$ & & & & $\begin{array}{l}\uparrow \mathrm{MD}^{* \circ \dagger}[88] \\
\downarrow \mathrm{FA}^{* \circ \dagger}[88]\end{array}$ & \\
\hline \multicolumn{6}{|c|}{ Infratentorial compartment } \\
\hline Whole & & $\begin{array}{l}\underset{\text { volume }}{\dagger}[54] \\
\uparrow \operatorname{MD}^{* \wedge \dagger}[81]\end{array}$ & $\begin{array}{l}\downarrow \text { volume }^{\dagger}[54] \\
\uparrow \mathrm{MD}^{* \wedge \dagger}[81]\end{array}$ & $\begin{array}{c}\downarrow \text { volume }{ }^{* \lambda \dagger} \\
{[55,57,60]} \\
\uparrow \mathrm{MCP} / \mathrm{SCP}^{* \circ}[37,54] \\
\downarrow \mathrm{M} / \mathrm{P}^{* \circ}[47,50,52] \\
\uparrow \mathrm{P}^{*} \mathrm{M}^{* \circ}[37,54] \\
\uparrow \mathrm{MRPI}^{* \circ} \\
{[37,50,52,54]} \\
\uparrow \mathrm{MD}^{* \dagger}[54,81] \\
\downarrow \mathrm{FA}^{*}[54]\end{array}$ & \\
\hline Midbrain & & & $\begin{array}{c}\downarrow \text { area }^{* \S}[49] \\
\downarrow \text { volume }^{\dagger}[59]\end{array}$ & $\begin{array}{c}\downarrow \text { area }{ }^{* \circ \uparrow} \uparrow[37,46,47, \\
49,52,54,88] \\
\downarrow \text { volume }^{* \circ \dagger}[59] \\
\uparrow \mathrm{ADC}^{* \#+\dagger}[73] \\
\uparrow \mathrm{MD}^{* \dagger \dagger}[88,89] \\
\downarrow \mathrm{FA}^{*}[54] \\
\downarrow \text { NAA/Cho }{ }^{\circ \dagger}[106]\end{array}$ & \\
\hline Substantia Nigra & $\begin{array}{c}\downarrow \mathrm{FA}^{*}[53] \\
\downarrow \operatorname{MTR}^{\dagger}[90]\end{array}$ & & & $\begin{array}{c}\uparrow \mathrm{MD}^{* \circ}[79] \\
\downarrow \mathrm{MTR}^{\dagger}[90] \\
\downarrow \text { SWI signal } \\
\text { intensity } \\
\text { *०† }[92]\end{array}$ & \\
\hline Red nucleus & & & $\begin{array}{c}\uparrow \text { phase shift values* } \\
{[95]}\end{array}$ & $\begin{array}{c}\uparrow \text { phase shift values* } \\
{[95]} \\
\downarrow \text { SWI signal } \\
\text { intensity }^{* 0 \dagger}[92]\end{array}$ & \\
\hline
\end{tabular}


TABLE 3: Continued.

\begin{tabular}{|c|c|c|c|c|c|}
\hline & MSA & MSA-C & MSA-P & PSP & CBS \\
\hline Pons & $\begin{array}{c}\downarrow \text { anteroposterior } \\
\text { diameter* }[53] \\
\downarrow \text { volume }{ }^{*}[53] \\
\uparrow \mathrm{ADC}^{* \wedge}[73] \\
\downarrow \mathrm{NAA} / \mathrm{Cr}^{\dagger}[105]\end{array}$ & $\begin{array}{l}\downarrow \text { area }^{* \wedge}[49] \\
\uparrow \operatorname{MD}^{* \wedge}[74]\end{array}$ & 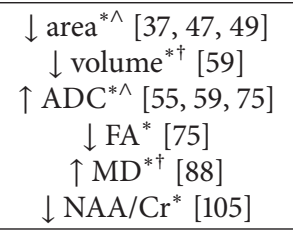 & $\downarrow$ area $^{*}[46,49,52,54]$ & \\
\hline Brainstem & & $\begin{array}{l}\underset{\text { volume }}{\dagger}[55] \\
\uparrow \operatorname{MD}^{* \wedge \dagger}[81]\end{array}$ & $\begin{array}{l}\downarrow \underset{\text { volume }}{* \dagger}[55,60] \\
\uparrow \mathrm{MD}^{* \wedge \dagger}[81]\end{array}$ & $\begin{array}{c}\uparrow \mathrm{MD}^{* \dagger}[54,55,81] \\
\downarrow \mathrm{FA}^{*}[54] \\
\downarrow \text { volume }^{*}[54] \\
\downarrow \text { NAA } / \mathrm{Cr}^{\dagger}[101]\end{array}$ & \\
\hline$S C P$ & & $\downarrow$ diameter ${ }^{*}[49]$ & $\uparrow \mathrm{MD}^{*}[89]$ & $\begin{array}{c}\downarrow \text { width }^{* \circ}[37] \\
\downarrow \text { diameter*॰ } \\
{[49,52,54]} \\
\downarrow \text { volume } \\
\uparrow \mathrm{ADC}^{* * \# \dagger}[59] \\
{[69,70,73,78]} \\
\uparrow \mathrm{MD}^{* \# \circ}[54,74,89] \\
\downarrow \mathrm{FA}^{*}[54,74]\end{array}$ & \\
\hline$M C P$ & $\begin{array}{l}\downarrow \text { mean width }{ }^{*}[53] \\
\uparrow \mathrm{MD}^{* \wedge}[53,74] \\
\downarrow \mathrm{FA}^{* \wedge \dagger}[53,74,85] \\
\uparrow \mathrm{ADC}^{* \wedge \dagger}[73,85]\end{array}$ & $\begin{array}{l}\downarrow \text { diameter }{ }^{* \wedge}[49] \\
\uparrow \operatorname{trace}(\mathrm{D})^{\circ \dagger}[71]\end{array}$ & $\begin{array}{c}\downarrow \text { width }^{* \circ}[37,48] \\
\downarrow \text { diameter* }[49] \\
\uparrow \operatorname{ADC}^{* \wedge \dagger}[48,67,77]\end{array}$ & $\begin{array}{c}\downarrow \text { diameter* } \\
{[49,52,54]} \\
\downarrow \mathrm{FA}^{* \# \dagger}[85] \\
\uparrow \mathrm{ADC}^{* \# \dagger}[85] \\
\end{array}$ & \\
\hline Cerebellum & $\begin{array}{l}\downarrow \text { volume }^{*}[53] \\
\uparrow \mathrm{ADC}^{* \wedge \dagger}[73] \\
\downarrow \downarrow \mathrm{FA}^{*}[53]\end{array}$ & $\begin{array}{c}\downarrow \text { volume }^{\dagger}[54] \\
\uparrow \operatorname{trace}(\mathrm{D})^{\circ \dagger}[71]\end{array}$ & $\begin{array}{c}\downarrow \text { volume }^{* \dagger} \\
{[54,59,60]} \\
\uparrow \text { ADC }^{*}[75] \\
\downarrow \text { FA }^{*}[75]\end{array}$ & $\begin{array}{c}\downarrow \text { volume }(\text { cortex })^{* \dagger} \\
{[60]}\end{array}$ & \\
\hline Cerebellar vermis & & $\uparrow \mathrm{MD}^{* \circ \dagger}[81]$ & $\uparrow \mathrm{MD}^{* \dagger}[81]$ & $\uparrow \mathrm{MD}^{* \dagger}[81]$ & \\
\hline $\begin{array}{l}\text { Cerebellar } \\
\text { hemispheres }\end{array}$ & & $\begin{array}{c}\uparrow \mathrm{MD}^{* \wedge \dagger}[81] \\
\downarrow \text { NAA/Cr }{ }^{* \wedge^{\circ}}[108]\end{array}$ & $\uparrow \mathrm{MD}^{* \wedge \dagger}[81]$ & $\begin{array}{c}\uparrow \mathrm{MD}^{*}[54] \\
\downarrow \text { NAA/Cr*广 }[108] \\
\downarrow \text { NAA/mI }{ }^{\dagger}[108]\end{array}$ & \\
\hline
\end{tabular}

ADC: apparent diffusion coefficient; MTR: magnetization transfer; rTrace(D): trace of diffusion tensor; NAA: N-acetyl-aspartate; CR: creatine; FA: fractional anisotropy; MD: mean diffusivity; SWI: susceptibility weighted imaging; HSR: hemispheric symmetry ratio; Cho: choline; CC: corpus callosum; RD: radial diffusivity; CST: corticospinal tract; DRTT: dentatorubrothalamic-tract; MCP: middle cerebellar peduncle; SCP: superior cerebellar peduncle; M: midbrain; P: pons; MRPI: MR parkinsonism index.

${ }^{*}$ versus PD; ${ }^{\wedge}$ versus PSP; ${ }^{\circ}$ versus MSA-P; ${ }^{\S}$ versus MSA-C; ${ }^{\#}$ versus MSA; ${ }^{\lambda}$ versus CBS; ${ }^{\dagger}$ versus HC.

using the same markers have demonstrated a highly variable diagnostic accuracy, with sensitivity and specificity ranging from $100 \%$ to much lower values (Table 2). Among all the techniques used in recent years, the simplest and most feasible in clinical practice seems to be the morphometric evaluation of midbrain, pons, cerebellar peduncles, and derived ratios, which has provided the best diagnostic accuracy values [37, 47-54]. However, a multimodal approach including parameters derived from different techniques may increase the global diagnostic accuracy [53].

A further important consideration is that in the vast majority of studies MR findings are compared between clinical, and not pathological, diagnoses, which may also be inaccurate [1]. Furthermore, most of the studies are on patients with long disease duration and it is uncertain whether the discriminating MRI findings would help the diagnosis in the early stage of the diseases.

These limitations notwithstanding, the in vivo identification of brain pathological patterns more frequently associated with different diseases (Table 3) is undoubtedly useful to better define a specific clinicoradiologic phenotype, which can increase the diagnostic probability of PD or MSA or PSP or CBS. Indeed, MR findings, by a qualitative or quantitative evaluation, can be useful mainly in those cases with an uncertain clinical phenotype. Specifically, qualitative/quantitative changes in the SN pars compacta indicate a degenerative parkinsonism. In this context, we propose the following conclusive considerations: (i) if no extranigral abnormalities are evident, the likelihood of PD diagnosis increases; (ii) if qualitative/quantitative changes are disclosed in the basal ganglia (mainly in the putamen), the likelihood of APS diagnosis increases; (iii) if qualitative/quantitative changes are disclosed in pons, MCPs, and cerebellum, the likelihood of MSA diagnosis increases; (iv) if qualitative/quantitative changes are disclosed in midbrain and SCPs, the likelihood of PSP diagnosis increases; (v) if qualitative/quantitative changes are disclosed in whole cerebral hemispheres (mainly in frontal and parietal cortices with asymmetric distribution), the likelihood of CBS diagnosis increases (Figure 5).

Taking into account the scientific literature, the feasibility in a clinical setting, and the availability of high-field MR scanners, a magnetic field of at least 1.5 Tesla is required by most 


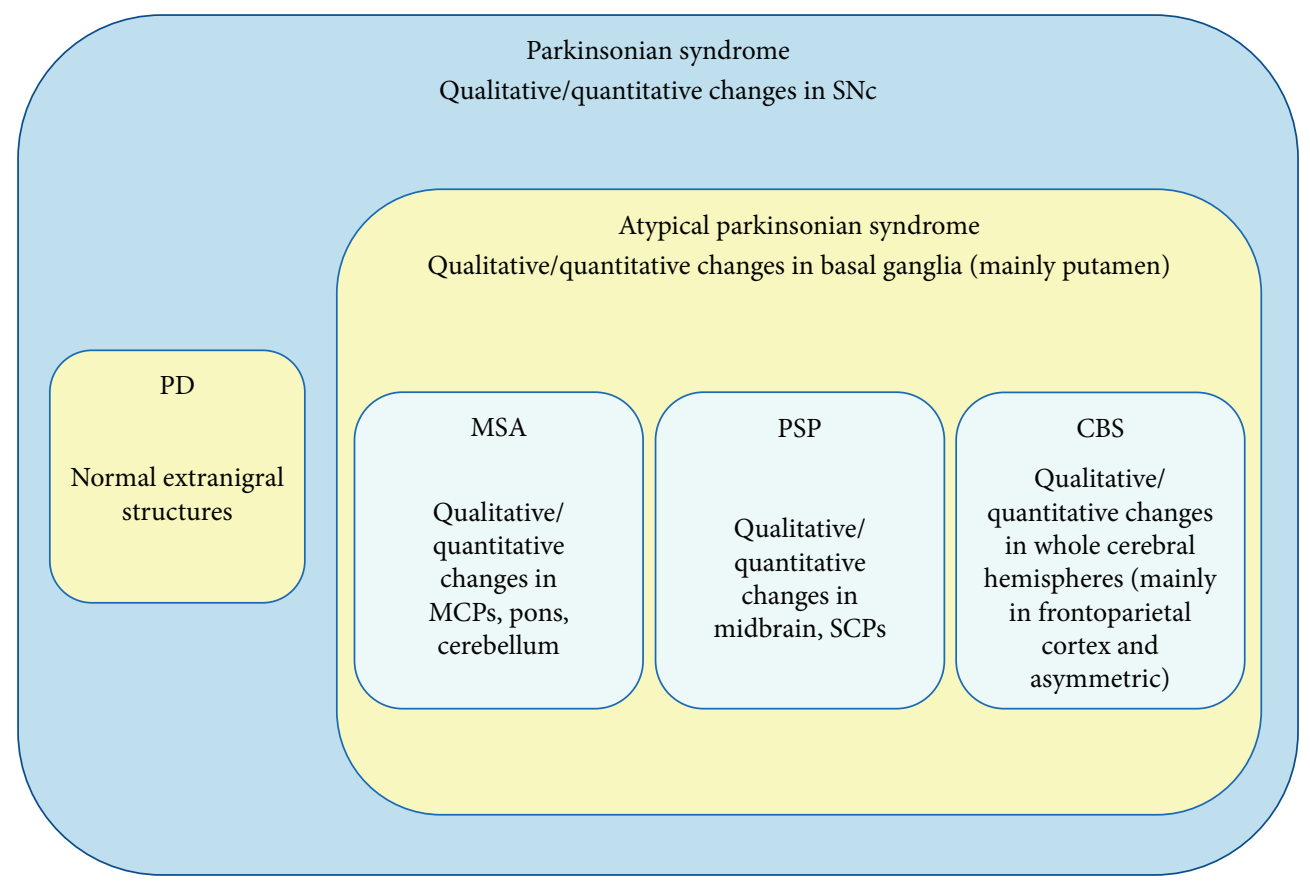

FIGURE 5: Flowchart of MR diagnosis in parkinsonian syndromes. SNC: substantia nigra pars compacta; PD: Parkinson's disease; MSA: Multiple System Atrophy; PSP: Progressive Supranuclear Palsy; CBS: Corticobasal Syndrome; MCPs: middle cerebellar peduncles; SCPs: superior cerebellar peduncles.

of the reported advanced techniques. Moreover, although a consensus on the type of sequences needed to discriminate PD from other mimics is lacking, an optimal protocol should include volumetric T1-weighted, axial T2-weighted fast spinecho/FLAIR, a diffusion-weighted sequence (DWI or better DTI), an iron-sensitive sequence, as $\mathrm{T} 2^{*}$-weighted or SWI, and ${ }^{1} \mathrm{H}$-MRS acquisition with a single-voxel localized within the basal ganglia and posterior fossa structures, that is, the pons or the cerebellar hemisphere.

In conclusion, MR markers are feasible and useful to increase the diagnostic accuracy of degenerative parkinsonian syndromes, although a rate of misdiagnosis remains currently inevitable. This emphasizes the need for specific biomarkers for the pathology underlying the different parkinsonian syndromes, for example, $\alpha$-synuclein or tau deposits, which along with a well-defined clinicoradiological phenotype can allow a more certain diagnosis. Such biomarkers can be biological, for example, from blood, liquor, skin, submandibular gland, or gut, but also imaging-based. Imaging biomarkers are currently obtained by the use of nuclear medicine tracers, such as amyloid or tau PET ligands, but in the future, similar tracers could be also available for MRI, for example, using nanoparticle-based contrast agents.

\section{Competing Interests}

The authors declare that they have no competing interests.

\section{Authors' Contributions}

G. Rizzo and S. Zanigni contributed equally to this work.

\section{Acknowledgments}

Cecilia Baroncini edited the English text.

\section{References}

[1] G. Rizzo, M. Copetti, S. Arcuti, D. Martino, A. Fontana, and G. Logroscino, "Accuracy of clinical diagnosis of Parkinson disease," Neurology, vol. 86, no. 6, pp. 566-576, 2016.

[2] S. Sharma, C. S. Moon, A. Khogali et al., "Biomarkers in Parkinson's disease (recent update)," Neurochemistry International, vol. 63, no. 3, pp. 201-229, 2013.

[3] M. Mascalchi, A. Vella, and R. Ceravolo, "Movement disorders: role of imaging in diagnosis," Journal of Magnetic Resonance Imaging, vol. 35, no. 2, pp. 239-256, 2012.

[4] S. T. Schwarz, M. Afzal, P. S. Morgan, N. Bajaj, P. A. Gowland, and D. P. Auer, "The 'swallow tail' appearance of the healthy nigrosome-a new accurate test of Parkinson's disease: a casecontrol and retrospective cross-sectional MRI study at 3T,' PLoS ONE, vol. 9, no. 4, Article ID e93814, 2014.

[5] P. Damier, E. C. Hirsch, Y. Agid, and A. M. Graybiel, "The substantia nigra of the human brain. II. Patterns of loss of dopamine-containing neurons in Parkinson's disease," Brain, vol. 122, no. 8, pp. 1437-1448, 1999.

[6] A. I. Blazejewska, S. T. Schwarz, A. Pitiot et al., "Visualization of nigrosome 1 and its loss in PD: pathoanatomical correlation and in vivo 7 T MRI," Neurology, vol. 81, no. 6, pp. 534-540, 2013.

[7] P. Gao, P. Y. Zhou, G. Li et al., "Visualization of nigrosomes1 in 3T MR susceptibility weighted imaging and its absence in diagnosing Parkinson's disease," European Review for Medical and Pharmacological Sciences, vol. 19, no. 23, pp. 4603-4609, 2015. 
[8] E. Reiter, C. Mueller, B. Pinter et al., "Dorsolateral nigral hyperintensity on 3.0T susceptibility-weighted imaging in neurodegenerative Parkinsonism," Movement Disorders, vol. 30, no. 8, pp. 1068-1076, 2015.

[9] L. Minati, M. Grisoli, F. Carella, T. De Simone, M. G. Bruzzone, and M. Savoiardo, "Imaging degeneration of the substantia Nigra in Parkinson disease with inversion-recovery MR imaging," American Journal of Neuroradiology, vol. 28, no. 2, pp. 309313, 2007.

[10] S. T. Schwarz, T. Rittman, V. Gontu, P. S. Morgan, N. Bajaj, and D. P. Auer, "T1-Weighted MRI shows stage-dependent substantia nigra signal loss in Parkinson's disease," Movement Disorders, vol. 26, no. 9, pp. 1633-1638, 2011.

[11] S. Reimão, P. Pita Lobo, D. Neutel et al., "Substantia nigra neuromelanin magnetic resonance imaging in de novo Parkinson's disease patients," European Journal of Neurology, vol. 22, no. 3, pp. 540-546, 2015.

[12] G. M. Halliday, J. L. Holton, T. Revesz, and D. W. Dickson, "Neuropathology underlying clinical variability in patients with synucleinopathies," Acta Neuropathologica, vol. 122, no. 2, pp. 187-204, 2011.

[13] T. Ozawa, D. Paviour, N. P. Quinn et al., "The spectrum of pathological involvement of the striatonigral and olivopontocerebellar systems in multiple system atrophy: clinicopathological correlations," Brain, vol. 127, no. 12, pp. 2657-2671, 2004.

[14] W. L. Lee, C.-C. Lee, W.-C. Shyu, P.-N. Chong, and S.-Z. Lin, "Hyperintense putaminal rim sign is not a hallmark of multiple system atrophy at 3T," American Journal of Neuroradiology, vol. 26, no. 9, pp. 2238-2242, 2005.

[15] G. Arabia, M. Morelli, S. Paglionico et al., "An magnetic resonance imaging $\mathrm{T} 2{ }^{*}$-weighted sequence at short echo time to detect putaminal hypointensity in Parkinsonisms," Movement Disorders, vol. 25, no. 16, pp. 2728-2734, 2010.

[16] A. Sugiyama, S. Ito, T. Suichi et al., "Putaminal hypointensity on T2* -weighted MR imaging is the most practically useful sign in diagnosing multiple system atrophy: a preliminary study," Journal of the Neurological Sciences, vol. 349, no. 1-2, pp. 174178, 2015.

[17] I. Hwang, C.-H. Sohn, K. M. Kang et al., "Differentiation of Parkinsonism-predominant multiple system atrophy from idiopathic Parkinson disease using 3T susceptibility-weighted MR imaging, focusing on putaminal change and lesion asymmetry," American Journal of Neuroradiology, vol. 36, no. 12, pp. 22272234, 2015.

[18] F. J. A. Meijer, A. van Rumund, B. A. C. M. Fasen et al., "Susceptibility-weighted imaging improves the diagnostic accuracy of 3T brain MRI in the work-up of Parkinsonism," American Journal of Neuroradiology, vol. 36, no. 3, pp. 454-460, 2015.

[19] D. R. Williams and A. J. Lees, "Progressive supranuclear palsy: clinicopathological concepts and diagnostic challenges," The Lancet Neurology, vol. 8, no. 3, pp. 270-279, 2009.

[20] D. W. Dickson, Z. Ahmed, A. A. Algom, Y. Tsuboi, and K. A. Josephs, "Neuropathology of variants of progressive supranuclear palsy," Current Opinion in Neurology, vol. 23, no. 4, pp. 394-400, 2010.

[21] R. M. Liscic, K. Srulijes, A. Gröger, W. Maetzler, and D. Berg, "Differentiation of progressive supranuclear palsy: clinical, imaging and laboratory tools," Acta Neurologica Scandinavica, vol. 127, no. 5, pp. 362-370, 2013.

[22] A. M. Grijalvo-Perez and I. Litvan, "Corticobasal degeneration," Seminars in Neurology, vol. 34, no. 2, pp. 160-173, 2014.
[23] F. J. A. Meijer, M. B. Aerts, W. F. Abdo et al., "Contribution of routine brain MRI to the differential diagnosis of parkinsonism: a 3-year prospective follow-up study," Journal of Neurology, vol. 259, no. 5, pp. 929-935, 2012.

[24] R. F. Delgado, P. R. Sanchez, H. Speckter et al., "Missense PANK2 mutation without 'eye of the tiger' sign: MR findings in a large group of patients with Pantothenate Kinase-Associated Neurodegeneration (PKAN)," Journal of Magnetic Resonance Imaging, vol. 35, no. 4, pp. 788-794, 2012.

[25] A. Stepens, I. Logina, V. Liguts et al., "A Parkinsonian syndrome in methcathinone users and the role of manganese," The New England Journal of Medicine, vol. 358, no. 10, pp. 1009-1017, 2008.

[26] D. A. Jacobs, C. E. Markowitz, D. S. Liebeskind, and S. L. Galetta, “The 'double panda sign' in Wilson's disease," Neurology, vol. 61, no. 7, p. 969, 2003.

[27] P. Singh, A. Ahluwalia, K. Saggar, and C. S. Grewal, "Wilson/s disease: MRI features," Journal of Pediatric Neurosciences, vol. 6, no. 1, pp. 27-28, 2011.

[28] G. Öz, J. R. Alger, P. B. Barker et al., "Clinical proton MR spectroscopy in central nervous system disorders," Radiology, vol. 270, no. 3, pp. 658-679, 2014.

[29] G. Rizzo, C. Tonon, D. Manners, C. Testa, and R. Lodi, "Imaging brain functional and metabolic changes in restless legs syndrome," Current Neurology and Neuroscience Reports, vol. 13, no. 9, article 372, 2013.

[30] E. M. Haacke, N. Y. C. Cheng, M. J. House et al., "Imaging iron stores in the brain using magnetic resonance imaging," Magnetic Resonance Imaging, vol. 23, no. 1, pp. 1-25, 2005.

[31] G. Bartzokis, M. Aravagiri, W. H. Oldendorf, J. Mintz, and S. R. Marder, "Field dependent transverse relaxation rate increase may be a specific measure of tissue iron stores," Magnetic Resonance in Medicine, vol. 29, no. 4, pp. 459-464, 1993.

[32] J. H. Jensen, R. Chandra, A. Ramani et al., "Magnetic field correlation imaging," Magnetic Resonance in Medicine, vol. 55, no. 6, pp. 1350-1361, 2006.

[33] R. J. Ogg, J. W. Langston, E. M. Haacke, R. G. Steen, and J. S. Taylor, "The correlation between phase shifts in gradient-echo MR images and regional brain iron concentration," Magnetic Resonance Imaging, vol. 17, no. 8, pp. 1141-1148, 1999.

[34] E. M. Haacke, Y. Xu, Y.-C. N. Cheng, and J. R. Reichenbach, "Susceptibility weighted imaging (SWI)," Magnetic Resonance in Medicine, vol. 52, no. 3, pp. 612-618, 2004.

[35] S. A. Smith, J. W. M. Bulte, and P. C. M. van Zijl, "Direct saturation MRI: theory and application to imaging brain iron," Magnetic Resonance in Medicine, vol. 62, no. 2, pp. 384-393, 2009.

[36] Y. Wang and T. Liu, "Quantitative susceptibility mapping (QSM): decoding MRI data for a tissue magnetic biomarker," Magnetic Resonance in Medicine, vol. 73, no. 1, pp. 82-101, 2015.

[37] A. Quattrone, G. Nicoletti, D. Messina et al., "MR imaging index for differentiation of progressive supranuclear palsy from Parkinson disease and the Parkinson variant of multiple system atrophy," Radiology, vol. 246, no. 1, pp. 214-221, 2008.

[38] J. Ashburner and K. J. Friston, "Voxel-based morphometry-the methods," NeuroImage, vol. 11, no. 6, pp. 805-821, 2000.

[39] G. Rizzo, C. Tonon, C. Testa et al., "Abnormal medial thalamic metabolism in patients with idiopathic restless legs syndrome," Brain, vol. 135, no. 12, pp. 3712-3720, 2012.

[40] B. Fischl, M. I. Sereno, R. B. H. Tootell, and A. M. Dale, "Highresolution intersubject averaging and a coordinate system for 
the cortical surface," Human Brain Mapping, vol. 8, no. 4, pp. 272-284, 1999.

[41] G. Rizzo, C. Tonon, M. L. Valentino et al., "Brain diffusionweighted imaging in Friedreich's ataxia," Movement Disorders, vol. 26, no. 4, pp. 705-712, 2011.

[42] L. L. Gramegna, C. Tonon, D. N. Manners et al., "Combined cerebellar proton MR spectroscopy and DWI study of patients with Friedreich's Ataxia," Cerebellum, pp. 1-7, 2016.

[43] S. M. Smith, M. Jenkinson, H. Johansen-Berg et al., "Tractbased spatial statistics: voxelwise analysis of multi-subject diffusion data," NeuroImage, vol. 31, no. 4, pp. 1487-1505, 2006.

[44] S. Jbabdi and H. Johansen-Berg, "Tractography: where do we go from here?” Brain Connectivity, vol. 1, no. 3, pp. 169-183, 2011.

[45] R. M. Henkelman, G. J. Stanisz, and S. J. Graham, "Magnetization transfer in MRI: a review," NMR in Biomedicine, vol. 14, no. 2, pp. 57-64, 2001.

[46] N. Kato, K. Arai, and T. Hattori, "Study of the rostral midbrain atrophy in progressive supranuclear palsy," Journal of the Neurological Sciences, vol. 210, no. 1-2, pp. 57-60, 2003.

[47] H. Oba, A. Yagishita, H. Terada et al., "New and reliable MRI diagnosis for progressive supranuclear palsy," Neurology, vol. 64, no. 12, pp. 2050-2055, 2005.

[48] G. Nicoletti, F. Fera, F. Condino et al., "MR imaging of middle cerebellar peduncle width: differentiation of multiple system atrophy from Parkinson disease," Radiology, vol. 239, no. 3, pp. 825-830, 2006.

[49] R. L. Gama, D. F. G. Távora, R. C. Bomfim, C. E. Silva, V. M. de Bruin, and P. F. de Bruin, "Morphometry MRI in the differential diagnosis of parkinsonian syndromes," Arquivos de Neuro-Psiquiatria, vol. 68, no. 3, pp. 333-338, 2010.

[50] A. Hussl, P. Mahlknecht, C. Scherfler et al., "Diagnostic accuracy of the magnetic resonance Parkinsonism index and the midbrain-to-pontine area ratio to differentiate progressive supranuclear palsy from Parkinson's disease and the Parkinson variant of multiple system atrophy," Movement Disorders, vol. 25, no. 14, pp. 2444-2449, 2010.

[51] G. Longoni, F. Agosta, V. S. Kostić et al., "MRI measurements of brainstem structures in patients with Richardson's syndrome, progressive supranuclear palsy-parkinsonism, and Parkinson's disease," Movement Disorders, vol. 26, no. 2, pp. 247-255, 2011.

[52] M. Morelli, G. Arabia, M. Salsone et al., "Accuracy of magnetic resonance parkinsonism index for differentiation of progressive supranuclear palsy from probable or possible Parkinson disease," Movement Disorders, vol. 26, no. 3, pp. 527-533, 2011.

[53] S. R. Nair, L. K. Tan, N. Mohd Ramli, S. Y. Lim, K. Rahmat, and H. Mohd Nor, "A decision tree for differentiating multiple system atrophy from Parkinson's disease using 3-T MR imaging," European Radiology, vol. 23, no. 6, pp. 1459-1466, 2013.

[54] S. Zanigni, G. Calandra-Buonaura, D. N. Manners et al., "Accuracy of MR markers for differentiating Progressive Supranuclear Palsy from Parkinson's disease," NeuroImage: Clinical, vol. 11, pp. 736-742, 2016.

[55] J. B. Schulz, M. Skalej, D. Wedekind et al., "Magnetic resonance imaging-based volumetry differentiates idiopathic Parkinson's syndrome from multiple system atrophy and progressive supranuclear palsy," Annals of Neurology, vol. 45, no. 1, pp. 6574, 1999.

[56] N. J. Cordato, C. Pantelis, G. M. Halliday et al., "Frontal atrophy correlates with behavioural changes in progressive supranuclear palsy," Brain, vol. 125, no. 4, pp. 789-800, 2002.
[57] K. Gröschel, T.-K. Hauser, A. Luft et al., "Magnetic resonance imaging-based volumetry differentiates progressive supranuclear palsy from corticobasal degeneration," NeuroImage, vol. 21, no. 2, pp. 714-724, 2004.

[58] D. C. Paviour, S. L. Price, J. M. Stevens, A. J. Lees, and N. C. Fox, "Quantitative MRI measurement of superior cerebellar peduncle in progressive supranuclear palsy," Neurology, vol. 64, no. 4, pp. 675-679, 2005.

[59] D. Paviour, S. L. Price, M. Jahanshahi, A. J. Lees, and N. C. Fox, "Regional brain volumes distinguish PSP, MSA-P, and PD: MRIbased clinico-radiological correlations," Movement Disorders, vol. 21, no. 7, pp. 989-996, 2006.

[60] D. Messina, A. Cerasa, F. Condino et al., "Patterns of brain atrophy in Parkinson's disease, progressive supranuclear palsy and multiple system atrophy," Parkinsonism and Related Disorders, vol. 17, no. 3, pp. 172-176, 2011.

[61] M. F. H. Schocke, K. Seppi, R. Esterhammer et al., "Diffusionweighted MRI differentiates the Parkinson variant of multiple system atrophy from PD," Neurology, vol. 58, no. 4, pp. 575-580, 2002.

[62] K. Seppi, M. F. H. Schocke, R. Esterhammer et al., "Diffusionweighted imaging discriminates progressive supranuclear palsy from $\mathrm{PD}$, but not from the parkinson variant of multiple system atrophy," Neurology, vol. 60, no. 6, pp. 922-927, 2003.

[63] M. F. H. Schocke, K. Seppi, R. Esterhammer et al., "Trace of diffusion tensor differentiates the Parkinson variant of multiple system atrophy and Parkinson's disease," NeuroImage, vol. 21, no. 4, pp. 1443-1451, 2004.

[64] K. Seppi, M. F. H. Schocke, E. Donnemiller et al., "Comparison of diffusion-weighted imaging and [123I]IBZM-SPECT for the differentiation of patients with the Parkinson variant of multiple system atrophy from those with Parkinson's disease," Movement Disorders, vol. 19, no. 12, pp. 1438-1445, 2004.

[65] K. Seppi, M. F. H. Schocke, K. Prennschuetz-Schuetzenau et al., "Topography of putaminal degeneration in multiple system atrophy: a diffusion magnetic resonance study," Movement Disorders, vol. 21, no. 6, pp. 847-852, 2006.

[66] G. Nicoletti, R. Lodi, F. Condino et al., "Apparent diffusion coefficient measurements of the middle cerebellar peduncle differentiate the Parkinson variant of MSA from Parkinson's disease and progressive supranuclear palsy," Brain, vol. 129, no. 10, pp. 2679-2687, 2006.

[67] D. C. Paviour, J. S. Thornton, A. J. Lees, and H. R. Jäger, "Diffusion-weighted magnetic resonance imaging differentiates Parkinsonian variant of multiple-system atrophy from progressive supranuclear palsy," Movement Disorders, vol. 22, no. 1, pp. 68-74, 2007.

[68] M. Köllensperger, K. Seppi, C. Liener et al., "Diffusion weighted imaging best discriminates PD from MSA-P: a comparison with tilt table testing and heart MIBG scintigraphy," Movement Disorders, vol. 22, no. 12, pp. 1771-1776, 2007.

[69] G. Nicoletti, C. Tonon, R. Lodi et al., "Apparent diffusion coefficient of the superior cerebellar peduncle differentiates progressive supranuclear palsy from Parkinson's disease," Movement Disorders, vol. 23, no. 16, pp. 2370-2376, 2008.

[70] G. Rizzo, P. Martinelli, D. Manners et al., "Diffusion-weighted brain imaging study of patients with clinical diagnosis of corticobasal degeneration, progressive supranuclear palsy and Parkinson's disease," Brain, vol. 131, no. 10, pp. 2690-2700, 2008.

[71] M. T. Pellecchia, P. B. Barone, C. Mollica et al., "Diffusionweighted imaging in multiple system atrophy: a comparison 
between clinical subtypes," Movement Disorders, vol. 24, no. 5, pp. 689-696, 2009.

[72] A. Umemura, T. Oeda, R. Hayashi et al., "Diagnostic accuracy of apparent diffusion coefficient and 123Imetaiodobenzylguanidine for differentiation of multiple system atrophy and Parkinson's disease," PLoS ONE, vol. 8, no. 4, Article ID e61066, 2013.

[73] K. Tsukamoto, E. Matsusue, Y. Kanasaki et al., "Significance of apparent diffusion coefficient measurement for the differential diagnosis of multiple system atrophy, progressive supranuclear palsy, and Parkinson's disease: evaluation by 3.0-T MR imaging," Neuroradiology, vol. 54, no. 9, pp. 947-955, 2012.

[74] C. R. V. Blain, G. J. Barker, J. M. Jarosz et al., "Measuring brain stem and cerebellar damage in parkinsonian syndromes using diffusion tensor MRI," Neurology, vol. 67, no. 12, pp. 2199-2205, 2006.

[75] M. Ito, H. Watanabe, Y. Kawai et al., "Usefulness of combined fractional anisotropy and apparent diffusion coefficient values for detection of involvement in multiple system atrophy," Journal of Neurology, Neurosurgery and Psychiatry, vol. 78, no. 7, pp. 722-728, 2007.

[76] S. Ito, T. Makino, W. Shirai, and T. Hattori, "Diffusion tensor analysis of corpus callosum in progressive supranuclear palsy," Neuroradiology, vol. 50, no. 11, pp. 981-985, 2008.

[77] E. J. Chung, E. G. Kim, J. S. Bae et al., "Usefulness of diffusionweighted MRI for differentiation between Parkinson's disease and Parkinson variant of multiple system atrophy," Journal of Movement Disorders, vol. 2, no. 2, pp. 64-68, 2009.

[78] A. Erbetta, M. L. Mandelli, M. Savoiardo et al., "Diffusion tensor imaging shows different topographic involvement of the thalamus in progressive supranuclear palsy and corticobasal degeneration," American Journal of Neuroradiology, vol. 30, no. 8, pp. 1482-1487, 2009.

[79] N. K. Focke, G. Helms, P. M. Pantel et al., "Differentiation of typical and atypical Parkinson syndromes by quantitative MR imaging," American Journal of Neuroradiology, vol. 32, no. 11, pp. 2087-2092, 2011.

[80] K. Boelmans, N. C. Bodammer, B. Suchorska et al., "Diffusion tensor imaging of the corpus callosum differentiates corticobasal syndrome from Parkinson's disease," Parkinsonism and Related Disorders, vol. 16, no. 8, pp. 498-502, 2010.

[81] G. Nicoletti, G. Rizzo, G. Barbagallo et al., "Diffusivity of cerebellar hemispheres enables discrimination of cerebellar or parkinsonian multiple system atrophy from progressive supranuclear palsy-Richardson syndrome and Parkinson disease," Radiology, vol. 267, no. 3, pp. 843-850, 2013.

[82] J. Prodoehl, H. Li, P. J. Planetta et al., "Diffusion tensor imaging of Parkinson's disease, atypical parkinsonism, and essential tremor," Movement Disorders, vol. 28, no. 13, pp. 1816-1822, 2013.

[83] S. Baudrexel, C. Seifried, B. Penndorf et al., "The value of putaminal diffusion imaging versus 18 -fluorodeoxyglucose positron emission tomography for the differential diagnosis of the Parkinson variant of multiple system atrophy," Movement Disorders, vol. 29, no. 3, pp. 380-387, 2014.

[84] C. P. Hess, C. W. Christine, A. C. Apple, W. P. Dillon, and M. J. Aminoff, "Changes in the thalamus in atypical Parkinsonism detected using shape analysis and diffusion tensor imaging," American Journal of Neuroradiology, vol. 39, no. 5, pp. 897-903, 2014.

[85] C. Nilsson, K. Markenroth Bloch, S. Brockstedt, J. Lätt, H. Widner, and E.-M. Larsson, "Tracking the neurodegeneration of parkinsonian disorders-a pilot study," Neuroradiology, vol. 49, no. 2, pp. 111-119, 2007.

[86] Y. Surova, F. Szczepankiewicz, J. Lätt et al., "Assessment of global and regional diffusion changes along white matter tracts in Parkinsonian disorders by MR tractography," PLoS ONE, vol. 8, no. 6, article e66022, 2013.

[87] J. Rosskopf, H.-P. Müller, H.-J. Huppertz, A. C. Ludolph, E. H. Pinkhardt, and J. Kassubek, "Frontal corpus callosum alterations in progressive supranuclear palsy but not in Parkinson's disease," Neurodegenerative Diseases, vol. 14, no. 4, pp. 184-193, 2014.

[88] Y. Surova, M. Nilsson, J. Lätt et al., "Disease-specific structural changes in thalamus and dentatorubrothalamic tract in progressive supranuclear palsy," Neuroradiology, vol. 57, no. 11, pp. 10791091, 2015.

[89] F. J. A. Meijer, A. van Rumund, A. M. Tuladhar et al., "Conventional 3T brain MRI and diffusion tensor imaging in the diagnostic workup of early stage parkinsonism," Neuroradiology, vol. 57, no. 7, pp. 655-669, 2015.

[90] T. Eckert, M. Sailer, J. Kaufmann et al., "Differentiation of idiopathic Parkinson's disease, multiple system atrophy, progressive supranuclear palsy, and healthy controls using magnetization transfer imaging," NeuroImage, vol. 21, no. 1, pp. 229-235, 2004.

[91] F. von Lewinski, C. Werner, T. Jörn, A. Mohr, F. Sixel-Döring, and C. Trenkwalder, "T2* -weighted MRI in diagnosis of multiple system atrophy. A practical approach for clinicians," Journal of Neurology, vol. 254, no. 9, pp. 1184-1188, 2007.

[92] D. Gupta, J. Saini, C. Kesavadas, P. S. Sarma, and A. Kishore, "Utility of susceptibility-weighted MRI in differentiating Parkinson's disease and atypical parkinsonism," Neuroradiology, vol. 52, no. 12, pp. 1087-1094, 2010.

[93] K. Boelmans, B. Holst, M. Hackius et al., "Brain iron deposition fingerprints in Parkinson's disease and progressive supranuclear palsy," Movement Disorders, vol. 27, no. 3, pp. 421-427, 2012.

[94] Y. Wang, S. R. Butros, X. Shuai et al., "Different iron-deposition patterns of multiple system atrophy with predominant parkinsonism and idiopathetic Parkinson diseases demonstrated by phase-corrected susceptibility-weighted imaging," American Journal of Neuroradiology, vol. 33, no. 2, pp. 266-273, 2012.

[95] Y.-H. Han, J.-H. Lee, B.-M. Kang et al., “Topographical differences of brain iron deposition between progressive supranuclear palsy and parkinsonian variant multiple system atrophy," Journal of the Neurological Sciences, vol. 325, no. 1-2, pp. 29-35, 2013.

[96] J.-H. Lee, Y.-H. Han, B.-M. Kang, C.-W. Mun, S.-J. Lee, and S.-K. Baik, "Quantitative assessment of subcortical atrophy and iron content in progressive supranuclear palsy and parkinsonian variant of multiple system atrophy," Journal of Neurology, vol. 260, no. 8, pp. 2094-2101, 2013.

[97] R. G. Yoon, S. J. Kim, H. S. Kim et al., "The utility of susceptibility-weighted imaging for differentiating Parkinsonism-predominant multiple system atrophy from Parkinson's disease: correlation with 18F-flurodeoxyglucose positron-emission tomography," Neuroscience Letters, vol. 584, pp. 296-301, 2015.

[98] C. A. Davie, G. K. Wenning, G. J. Barker et al., "Differentiation of multiple system atrophy from idiopathic Parkinson's disease using proton magnetic resonance spectroscopy," Annals of Neurology, vol. 37, no. 2, pp. 204-210, 1995.

[99] F. Federico, I. L. Simone, V. Lucivero et al., "Proton magnetic resonance spectroscopy in Parkinson's disease and progressive 
supranuclear palsy," Journal of Neurology, Neurosurgery and Psychiatry, vol. 62, no. 3, pp. 239-242, 1997.

[100] F. Federico, I. L. Simone, V. Lucivero et al., "Proton magnetic resonance spectroscopy in Parkinson's disease and atypical parkinsonian disorders," Movement Disorders, vol. 12, no. 6, pp. 903-909, 1997.

[101] G. Tedeschi, I. Litvan, S. Bonavita et al., "Proton magnetic resonance spectroscopic imaging in progressive supranuclear palsy, Parkinson's disease and corticobasal degeneration," Brain, vol. 120, no. 9, pp. 1541-1552, 1997.

[102] F. Federico, I. L. Simone, V. Lucivero et al., "Usefulness of proton magnetic resonance spectroscopy in differentiating parkinsonian syndromes," The Italian Journal of Neurological Sciences, vol. 20, no. 4, pp. 223-229, 1999.

[103] K. Abe, H. Terakawa, M. Takanashi et al., "Proton magnetic resonance spectroscopy of patients with parkinsonism," Brain Research Bulletin, vol. 52, no. 6, pp. 589-595, 2000.

[104] C. E. Clarke and M. Lowry, "Basal ganglia metabolite concentrations in idiopathic Parkinson's disease and multiple system atrophy measured by proton magnetic resonance spectroscopy," European Journal of Neurology, vol. 7, no. 6, pp. 661-665, 2000.

[105] H. Watanabe, H. Fukatsu, and M. Katsuno, "Multiple regional 1H-MR spectroscopy in multiple system atrophy: NAA/Cr reduction in pontine base as a valuable diagnostic marker," Journal of Neurology, Neurosurgery and Psychiatry, vol. 75, no. 1, pp. 103-109, 2004.

[106] L. F. R. Vasconcellos, S. A. Pereira Novis, D. M. Moreira, A. L. Z. Rosso, and A. C. C. B. Leite, "Neuroimaging in Parkinsonism: a study with magnetic resonance and spectroscopy as tools in the differential diagnosis," Arquivos de Neuro-Psiquiatria, vol. 67, no. 1, pp. 1-6, 2009.

[107] C. A. Guevara, C. R. Blain, D. Stahl, D. J. Lythgoe, P. N. Leigh, and G. J. Barker, "Quantitative magnetic resonance spectroscopic imaging in Parkinson's disease, progressive supranuclear palsy and multiple system atrophy," European Journal of Neurology, vol. 17, no. 9, pp. 1193-1202, 2010.

[108] S. Zanigni, C. Testa, G. Calandra-Buonaura et al., "The contribution of cerebellar proton magnetic resonance spectroscopy in the differential diagnosis among parkinsonian syndromes," Parkinsonism and Related Disorders, vol. 21, no. 8, pp. 929-937, 2015.

[109] W. R. W. Martin, M. Wieler, and M. Gee, "Midbrain iron content in early Parkinson disease: a potential biomarker of disease status," Neurology, vol. 70, no. 16, pp. 1411-1417, 2008.

[110] P. Péran, A. Cherubini, F. Assogna et al., "Magnetic resonance imaging markers of Parkinson's disease nigrostriatal signature," Brain, vol. 133, no. 11, pp. 3423-3433, 2010.

[111] A. Gröger, B. Bender, I. Wurster, G. L. Chadzynski, U. Klose, and D. Berg, "Differentiation between idiopathic and atypical parkinsonian syndromes using three-dimensional magnetic resonance spectroscopic imaging," Journal of Neurology, Neurosurgery and Psychiatry, vol. 84, no. 6, pp. 644-649, 2013.

[112] D. E. Vaillancourt, M. B. Spraker, J. Prodoehl et al., "Highresolution diffusion tensor imaging in the substantia nigra of de novo Parkinson disease," Neurology, vol. 72, no. 16, pp. 13781384, 2009.

[113] J. Langley, D. E. Huddleston, M. Merritt et al., "Diffusion tensor imaging of the substantia nigra in Parkinson's disease revisited," Human Brain Mapping, vol. 3, no. 7, pp. 2547-2556, 2016.

[114] D. K. Jones and M. Cercignani, "Twenty-five pitfalls in the analysis of diffusion MRI data," NMR in Biomedicine, vol. 23, no. 7, pp. 803-820, 2010. 


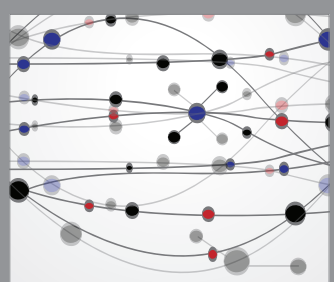

The Scientific World Journal
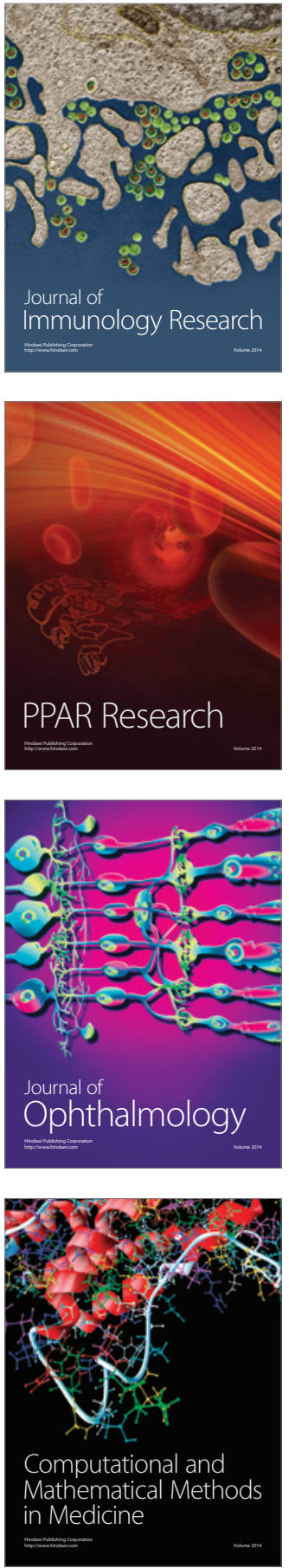

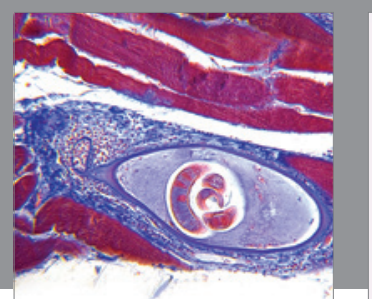

Gastroenterology Research and Practice

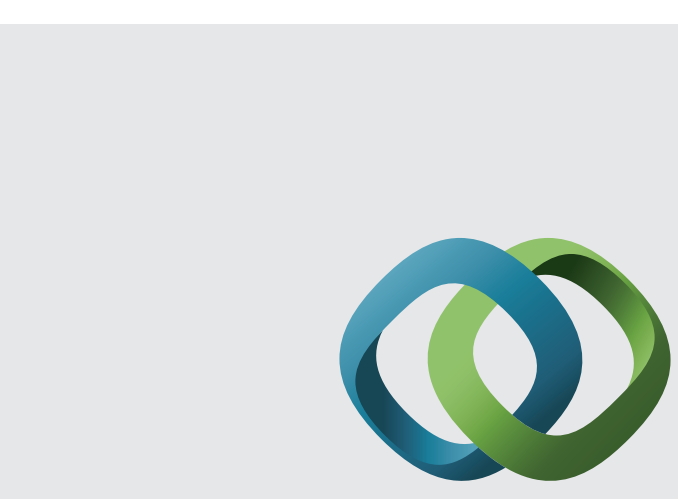

\section{Hindawi}

Submit your manuscripts at

http://www.hindawi.com
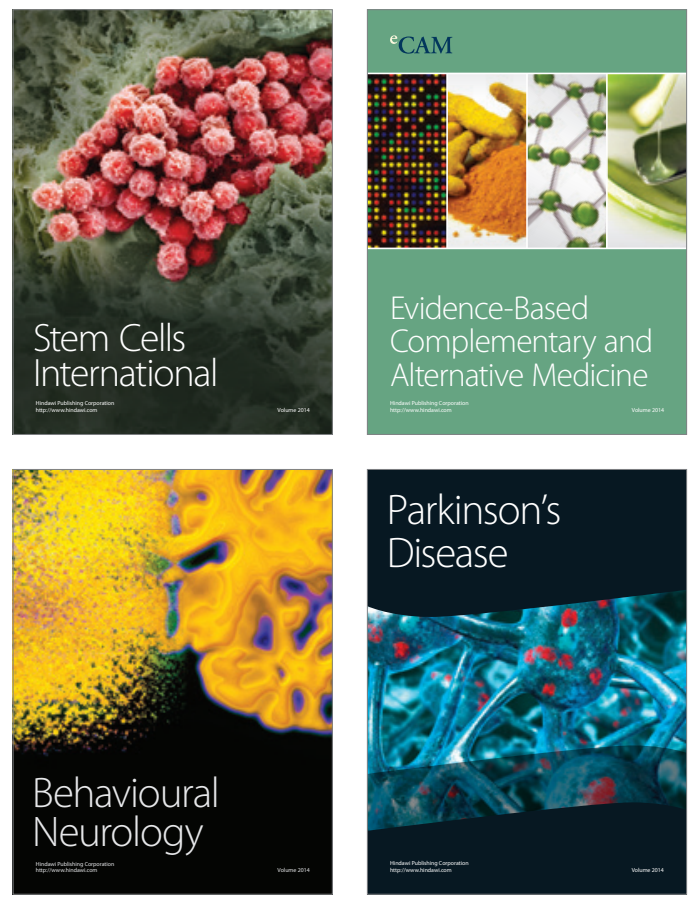
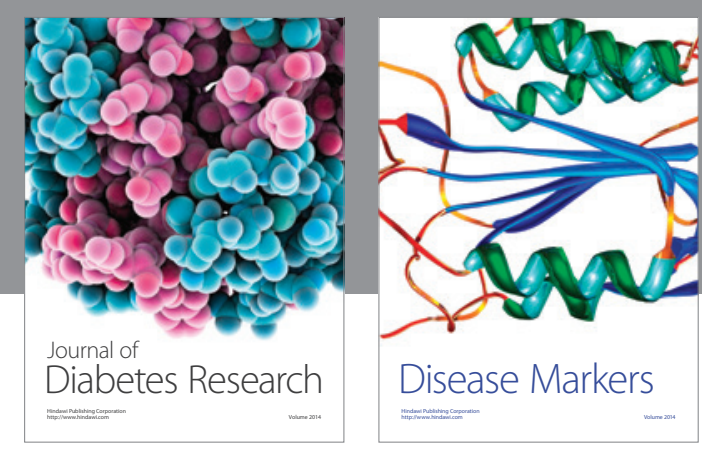

Disease Markers
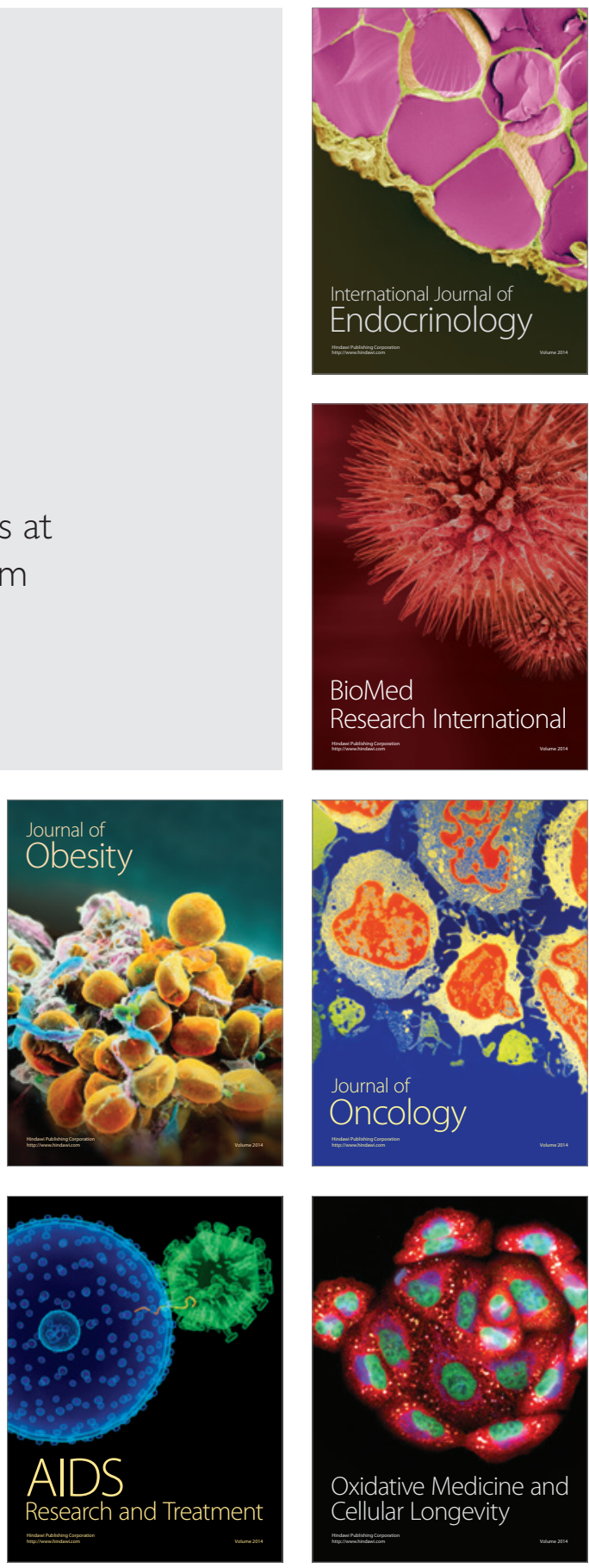